\title{
Instructive starPEG-Heparin biohybrid 3D cultures for modeling human neural stem cell plasticity, neurogenesis, and neurodegeneration
}

Christos Papadimitriou ${ }^{1,2}$, Mehmet I. Cosacak ${ }^{1,2}$, Violeta Mashkaryan ${ }^{1,2}$, Hilal Celikkaya ${ }^{1,2}$, Laura Bray ${ }^{3,4}$, Prabesh Bhattarai ${ }^{1,2}$, Heike Hollak ${ }^{1,2}$, Xin Chen $^{5}$, Shuijin $\mathrm{He}^{5}$, Christopher L. Antos ${ }^{5,6}$, Alvin K. Thomas ${ }^{7}$, Jens Friedrichs ${ }^{2,3}$, Andreas Dahl ${ }^{7}$, Yixin Zhang ${ }^{8}$, Uwe Freudenberg ${ }^{2,3}$, Carsten Werner $^{2,3}$, Caghan Kizil ${ }^{1,2, *}$

1 German Center for Neurodegenerative Diseases (DZNE) Dresden, Helmholtz Association, Arnoldstr. 18, 01307, Dresden, Germany.

132 Center for Regenerative Therapies (CRTD), Technische Universität Dresden, Fetscherstr. 105, 01307, Dresden, Germany.

$15{ }^{3}$ Leibniz Institute of Polymer Research Dresden, Max Bergmann Center of 16 Biomaterials Dresden, Hohe Str. 6, 01069, Dresden, Germany.

$17{ }^{4}$ Institute of Health Biomedical Innovation (IHBI), Queensland University 18 of Technology, 60 Musk Avenue, Kelvin Grove, 4059, Australia.

195 School of Life Sciences and Technology, ShanghaiTech University, 20 Shanghai 201210, People's Republic of China.

216 Institut für Pharmakologie und Toxikologie, Technische Universität 22 Dresden Medizinische Fakultät, Fetscherstr. 74, 01307, Dresden, 23 Germany.

$24{ }^{7}$ Deep Sequencing Group, Biotechnology Center, TU Dresden, Fetscherstr. 25 105, 01307, Dresden, Germany.

$26{ }^{8}$ B CUBE, Center for Molecular Bioengineering, TU Dresden, Arnoldstr. 18, 27 10307, Dresden, Germany. 
Keywords:

Human neural stem cell, plasticity, Amyloid-beta42, whole transcriptome sequencing, 3D culture, neurogenesis

\section{Abstract}

Three-dimensional models of human neural development and neurodegeneration are crucial when exploring stem-cell-based regenerative therapies in a tissue-mimetic manner. However, existing 3D culture systems are not sufficient to model the inherent plasticity of NSCs due to their ill-defined composition and lack of controllability of the physical properties. Adapting a glycosaminoglycan-based, cell-responsive hydrogel platform, we stimulated primary and induced human neural stem cells (NSCs) to manifest neurogenic plasticity and form extensive neuronal networks in vitro. The 3D cultures exhibited neurotransmitter responsiveness, electrophysiological activity, and tissue-specific extracellular matrix (ECM) deposition. By whole transcriptome sequencing, we identified that 3D cultures express mature neuronal markers, and reflect the in vivo make-up of mature cortical neurons compared to 2D cultures. Thus, our data suggest that our established 3D hydrogel culture supports the tissue-mimetic maturation of human neurons. We also exemplarily modeled neurodegenerative conditions by treating the cultures with $A \beta 42$ peptide and observed the known human pathological effects of Alzheimer's disease including reduced NSC proliferation, impaired neuronal network formation, synaptic loss and failure in ECM deposition as well as elevated Tau hyperphosphorylation and formation of neurofibrillary tangles. We determined the changes in transcriptomes of primary and induced NSC-derived neurons after $A \beta 42$, providing a useful resource for further studies. Thus, our hydrogel-based human cortical 3D cell culture is a powerful platform for studying various aspects of neural development and neurodegeneration, as exemplified for A $\beta 42$ toxicity and neurogenic stem cell plasticity. 


\section{Significance}

Neural stem cells (NSC) are reservoir for new neurons in human brains, yet they fail to form neurons after neurodegeneration. Therefore, understanding the potential use of NSCs for stem cell-based regenerative therapies requires tissue-mimetic humanized experimental systems. We report the adaptation of a $3 D$ bio-instructive hydrogel culture system where human NSCs form neurons that later form networks in a controlled microenvironment. We also modeled neurodegenerative toxicity by using Amyloid-beta4 peptide, a hallmark of Alzheimer's disease, observed phenotypes reminiscent of human brains, and determined the global gene expression changes during development and degeneration of neurons. Thus, our reductionist humanized culture model will be an important tool to address NSC plasticity, neurogenicity, and network formation in health and disease.

\section{Text}

\section{Introduction}

Human brain development and neuronal diseases cannot be modeled adequately by current animal models (LaFerla and Green, 2012); therefore, the development of novel humanized systems that manifest neurogenic plasticity is necessary. The brain's plasticity provides an endogenous reservoir of cells that could be harnessed to physiologically enhance brain capacity or for neuronal repair (Gage and Temple, 2013; Wyss-Coray, 2016). Therefore, it is fundamentally important to understand how neurons develop to form a hard-wired network, how new networks are generated when newly formed neurons are incorporated, and how stem cells contribute to these processes. Animal models and cell culture experiments examining how mammalian brains develop and elucidating the molecular programs regulating this process have been invaluable (Molyneaux et al., 2007). However, the human brain might exhibit differences compared with the model systems that might not be discernable with existing tools. 
97 The human brain cannot repair the loss of neurons caused by 98 neurodegenerative diseases (ND), in part due to reduced stem cell 99 proliferation and neurogenesis (Tincer et al., 2016). These combinatorial 100 effects exacerbate the manifestation of the disease. In ND states in 101 humans, overall plasticity is severely decreased (Heneka et al., 2015; 102 Nalbantoglu et al., 1997; Selkoe, 2002), however, we know little about 103 how to circumvent this reduction due to lack of appropriate experimental 104 systems. Alzheimer's disease (AD) is the most common ND, and one of its 105 hallmarks is the aggregation of amyloid protein cleavage products 106 mainly amyloid- $\beta$-42 (A 42 ) peptides (Esler and Wolfe, 2001; Haass and 107 Selkoe, 2007; Nalbantoglu et al., 1997). The disease state produces an 108 inhospitable environment lacking homeostasis in which stem cells are 109 unable to form neurons and new cells do not survive and successfully 110 integrate into existing circuitry. Therefore, understanding stem cell 111 plasticity and neuronal behavior in disease-related settings is critical to 112 determine if a stem cell can regain proliferative and neurogenic function 113 or whether a newborn neuron can survive and integrate into the 114 remaining circuitry despite prevalent amyloid toxicity in the brain.

115 The overall plasticity of the human brain requires neural stem cell (NSC) 116 proliferation, neurogenesis and neuronal network formation (Alvarez117 Buylla et al., 2002; Gage, 2000). However, although NSCs in human brain 118 possess the plasticity to fulfill all these steps, 2D culture conditions are 119 insufficient to generate the connected arbors and long-term behaviors 120 observed in the brain (Haycock, 2011; Justice et al., 2009). Culture 121 conditions that drive the generation of neurons that retain a mature 122 neuronal morphology and form synapses and 3D patterns are needed to 123 address how the entire spectrum of plasticity manifests in the human 124 brain. In addition, paracrine effects from the interplay between glia and 125 neurons, the nascent cellular microenvironment, and the extracellular 126 matrix (ECM) regulate cell fate decisions (Lutolf et al., 2009). In 127 particular, the modulation of mechanical cues, the degradability of the 128 matrix, and the administration of soluble effectors is known to control 129 stem cell fate (Discher et al., 2009). Therefore, we require advanced 130 customized and controllable assay systems for human neural stem cells. 
131 Animal models of $A D$ are unable to recapitulate the entire human disease 132 spectrum (LaFerla and Green, 2012), suggesting that human cells might 133 have a different physiological response than animal cells in response to 134 NDs. Additionally, revolutionary 3D technologies are useful tools for 135 addressing specific questions; however, they require highly complex 136 culture conditions, are difficult to establish and reproduce, and the 137 content of the scaffolds often have unintended consequences on the 138 encapsulated cells. Therefore, in vitro systems utilizing human cells to 139 model neural development or neurodegeneration in an in vivo-like 3D 140 environment that is amenable to manipulation and monitoring would be 141 highly beneficial. Therefore, in this study, we adapted a highly tunable 142 and defined glycosaminoglycan (GAG)-based 3D matrix system (Chwalek 143 et al., 2014; Maitz et al., 2013) to culture primary and induced human 144 neural stem cells, which manifest their plasticity and neurogenic capacity. 145 This system allowed us to systematically vary the local cellular 146 environment in terms of stiffness, degradability and presentation of GAG147 affine signaling molecules (Capilla and Linhardt, 2002) to identify optimal 148 conditions that reproducibly form networks of mature neurons and glia 149 that serve as neural stem cells. We also recapitulated important aspects 150 of $A D$ by modeling amyloid toxicity. $A \beta 42$ treatment impaired network 151 formation and progenitor cell proliferation and induced human 152 pathological hallmarks, including Tau hyperphosphorylation and 153 neurofibrillary tangle formation, suggesting that our biohybrid 3D culture 154 system can be used to address questions regarding neural stem cell 155 proliferation, neurodevelopment and neurodegenerative diseases in a 156 reductionist and tissue mimetic pre-clinical setting.

\section{Results}

158 To generate a culture system that would allow NSCs to manifest their 159 plasticity and neurogenic capacity in a tissue-representative manner, we 160 applied a modular biohybrid material based on star-shaped poly(ethylene 161 glycol) (starPEG) and GAG heparin (HEP) that can be used to 162 independently tune mechanical cues and biomolecular functionalization 163 (Freudenberg et al., 2012; Tsurkan et al., 2013) when embedding primary 164 human neural stem cells (human cortical astrocytes, from here on primary 
165 NSCs) derived from fetal tissue at gestation week 21 (Fig. 1A). We 166 systematically varied the biohybrid matrix in terms of stiffness, cell167 responsive remodeling potential, and the presence of soluble effector 168 mediating GAGs to induce the cellular morphology reminiscent of in vivo 169 (Supplementary Figure 1). After varying the stiffness (Young's modulus) 170 of the hydrogels from $0.5 \mathrm{kPa}$ to $3 \mathrm{kPa}$ (Fig. $1 \mathrm{~B}, \mathrm{~B}^{\prime}$ ), $1.2 \mathrm{kPa}$ was the 171 optimal stiffness to promote the formation of extended neuronal networks 172 by primary NSCs (Fig. 1B'). Although soft gels (0.5 kPa) disintegrated 173 within 1 week (data not shown), stiff gels ( $3 \mathrm{kPa}$ ) resulted in round non174 proliferative cells that do not grow into the matrix or form a network (Fig. $\left.1751 B^{\prime}\right)$.

176 Tissue characteristics and patterning can be influenced by the cell177 responsive remodeling of biohybrid hydrogels, which can be achieved by 178 the incorporation of matrix-metalloprotease-cleavable linkers (Chwalek et 179 al., 2011; Tsurkan et al., 2010). Using MMP-cleavable hydrogels (starPEG180 MMP-HEP) (Fig. 1C, $C^{\prime}$ ), directly linked starPEG-HEP gels (Fig. 1D, $D^{\prime}$ ) and 181 non-degradable controls with a scrambled (MMP-insensitive) peptide 182 sequence (starPEG-scr-HEP) (Fig. 1E, $E^{\prime}$ ), we found that only MMP183 responsive hydrogels induced the formation of a neuronal network (Fig. 184 1C). Given that the optimal stiffness was 1200 Pa for all of these gels (Fig. $1851 \mathrm{~F}$ ), these results clearly point to enzymatic remodeling involving MMPs 186 (Agrawal et al., 2008) as a crucial process for axodendritic outgrowth and 187 the formation of mature arbors. Moreover, if an enzymatically degradable 188 hydrogel of inert starPEG was formed, the cells did not proliferate and did 189 not display axodendritic extensions, highlighting the importance of a 190 factor regulating GAG-heparin interaction within the matrices (data not 191 shown). Because heparin can bind multiple insoluble matrix proteins (e.g., 192 laminin, fibronectin) and other soluble growth factors (Capila and 193 Linhardt, 2002; Garg et al., 2011), it might mediate the activity and 194 presentation of cell-secreted molecules and thus indirectly control cell fate 195 processes. Sulfated GAGs, particularly heparan sulfate and chondroitin 196 sulfate, are well known to be a major component of neuronal ECMs and 197 are crucial for developmental processes and axon guidance (Lau et al., 198 2013). Thus, the MMP-cleavable heparin hydrogel matrix provided optimal 
199 conditions with respect to initial stiffness and the presence of sulfated 200 GAGs and promoted remodeling in a well-orchestrated and timely way via 201 MMP-sensitive peptide linkers.

202 Since the development of 3D cultures requires MMP-cleavage, we 203 hypothesized that the observed maturation and patterning might follow a 204 replacement of the initial scaffold with the cells' own matrix. Therefore, 205 we immunostained the cultures for fibronectin and laminin to test this 206 hypothesis and found that the 3D gels generate fibronectin (Fig. 1G,H) 207 and laminin (Fig. 1I,J) de novo, suggesting that neural stem cells and 208 neurons remodel the matrix allowing to generate a liberal stem cell niche 209 according to the needs of the NSCs. To investigate whether this 210 remodeling would alter the stiffness of the gel matrix, we performed 211 atomic force microscopy analyses of the gels at different time points and 212 observed that the initial elastic modulus of $1.3 \mathrm{kPa}$ is reduced gradually to $2130.3 \mathrm{kPa}$ after 14 days (Fig. $1 \mathrm{~K}$ ), indicating that the development of 214 starPEG-Heparin 3D cultures generate human brain-mimetic physical 215 properties. This is particularly important because widely used 3D scaffolds 216 (e.g.: Matrigel) cannot be regulated in their stiffness by the encapsulated 217 cells, and therefore they deviate from tissue-mimetic physical features. 218 Indeed, when we compared the network forming ability of primary human 219 NSCs in identical culture conditions in starPEG-Heparin and Matrigel, we 220 found that the neurogenic capacity and network-formation ability of 221 primary NSCs manifest significantly better in starPEG-Heparin gels 222 (Supplementary Figure 2), indicating that starPEG-Heparin composition 223 favors an unprecedented tissue-mimetic environment for primary NSCs.

224 Based on our cell-matrix interaction results, we hypothesized that the 225 maturation of neuronal networks (Fig. 1L) might resemble human 226 neurodevelopment. We analyzed the gels at various time points to 227 address this hypothesis (Fig. 1M-P). One week after seeding, the gel 228 contained sparsely distributed GFAP-positive glia with a 3D arborized 229 morphology (Fig. 1M). After two weeks of culture, we started to observe 230 acetylated tubulin-positive neurons extending processes and organizing 231 themselves into clusters (Fig. 1N; Suppl. Video 1). At 3 weeks, the 232 cultures produced an extensive and elaborate network of neurons with 
233 interspersed glia (Fig. 10) in close association with neurons (Fig. 1P). We 234 also determined that the primary NSC cultures expressed neural stem cell 235 markers such as SOX2 and GFAP, and neural fate determinants such as 236 NEUROD in spatially overlapping, but distinct domains (Fig. 1Q, X-axis 237 view; Fig. 1R, Z-axis view), indicating that neural stem cell plasticity 238 programs and neurogenic activity drive the development of the neuronal 239 networks, and the stem cell compartments may pattern in a 3D topology 240 similar to human brains.

241 Based on our findings, we hypothesized that 3D cultures would provide a 242 superior 3D topological environment to pNSCs, and therefore would 243 instruct a gene expression profile that would be closer to in vivo. To 244 investigate how 3D cultures would molecularly differ from 2D cultures, we 245 cultured primary NSCs in 2D and 3D using identical culture conditions for 246 three weeks. After isolating total mRNA, we performed whole 247 transcriptome sequencing, and observed that a considerable number of 248 genes are differentially expressed between 2D and 3D cultures (Fig. 2A, 249 Supplementary Dataset 1 ). In order to identify the pathways and 250 molecular programs represented better in 3D cultures, we performed 251 pathway and enrichment analyses (Fig. 2B). We found that 3D cultures 252 express genes related to several pathways that characterize mature 253 neuronal physiology such as focal adhesion, ECM-receptor interaction, 254 axon guidance, and various signaling pathways (Fig. 2B, Suppl. Dataset 255 2). Additionally, cellular component analyses indicate that the 3D cultures 256 express the genes, the protein products of which are related to various 257 mature neuronal processes such as synapses and axons (Fig. 2B). These 258 results indicate that our hydrogel cultures provide the 3D topology and 259 instructive environment to generate neuronal networks from primary 260 human NSCs in a tissue-mimetic manner, which is not the case for 2D 261 cultures.

262 To verify that the 3D cultures of primary human NSCs generate mature 263 neurons resembling the in vivo conditions, we immunostained 3D cultures 264 for the synaptic marker Synaptophysin (Fig. 2C), which clusters at 265 neuronal junctions (Fig. 2D) and boutons (Fig. 2E), and generate pre- and 266 post-synaptic termini (Fig. 2F), indicating that neurons in 3D cultures 
267 develop enough to form synaptic connections, which we did not observe in 268 2D conditions (data not shown). We found that the neurons formed in the 269 3D starPEG-HEP-based hydrogels were also expressing neurotransmitter 270 receptors such as VGLUT1 (Fig. 2G), and are responsive to 271 neurotransmitters such as glutamate, as shown by increased intracellular 272 calcium levels (Fig. 2H-J; Suppl. Video 2) following the transfection of 273 plasmids expressing the GCamP6f calcium sensor driven by the CMV 274 promoter. We also performed electrophysiology experiments assessing 275 sodium and potassium channel activity to determine whether the cells 276 from the matrices were functional neurons. We observed potassium and 277 sodium channel activity as well as spontaneous firing of neurons in whole 278 cell patch clamp recordings (Fig. $2 \mathrm{~K}$ ), indicating that cells cultured within 279 the hydrogels differentiated into functionally active neurons. These results 280 indicate that starPEG-HEP-hydrogel-based 3D cultures of primary human 281 NSCs are able to generate an elaborate and mature network of neurons 282 and glia in a three dimensional organization.

283 To determine the cortical subtypes produced in 3D and 2D conditions, we 284 compared the expression levels of a selected set of cortical marker genes 285 in relation to their spatial confinement to different layers (Fig. 2L; from 286 (Molyneaux et al., 2007)). We observed that 3D conditions favor for 287 expression of a larger and more complete set of cortical marker genes 288 especially associated with layers IV, V and VI, compared to 2D (Fig. 2L). 289 Similarly, although 2D and 3D conditions allow expression of NSC markers 290 at high levels, the major cortical pro-neural gene ASCL1 is expressed in 291 rather low levels in 2D conditions while 3D cultures allow significantly 292 higher levels of ASCL1 expression (Fig. 2M). To validate our deep 293 sequencing results and heat map analyses, we performed 294 immunohistochemistry for pro-neural determinant ASCL1 and early 295 neuronal marker DCX, cortical layer markers SATB2, CTIP2, Reelin 296 (RELN), DBX1, FOXO1, FOXP2, CRYM, and mature neuronal markers 297 Neurofilament-200 and NeuN (Fig. 2N). We found that 3D cultures allow 298 expression of pro-neural fate determinants, and production of different 299 lineage subtypes of cortical neurons (Fig. 2N). 
300 To test whether we could use our system with other types of neural stem

301 cells, we cultured iPSC-derived human neural stem cells (iNSCs) in culture 302 under conditions identical to those of primary NSCs (pNSCs), and 303 observed extensive neurogenesis and network formation similar to pNSCs 304 at 3 weeks of cultures (Fig. 3A,B, Suppl. Movie 3), suggesting that our 305 instructive hydrogel composition can support development of networks 306 from various types of neural stem cell sources.

307 To compare the molecular expression profiles of pNSC and iNSC cultures, 308 we isolated total RNA and performed whole transcriptome sequencing on 309 iNSCs and compared the reads to those of the cultures with pNSCs (Fig. 3103 3, Suppl. Dataset 3). We found that although both iNSCs and pNSCs can 311 generate neuronal networks in 3D cultures, these two cell types differ 312 significantly in their gene expression patterns (Fig. 3C; Suppl. Dataset 4). 313 Interestingly, we found that compared to iNSCs, pNSC cultures express 314 genes related to extracellular matrix and plasma membrane more (Fig. 315 3D; Suppl. Dataset 5), and these differences enrich pathways such as 316 various signaling pathways, axon guidance, neuroactive ligand-receptor 317 interaction and metabolism (Fig. 3D; Suppl. Dataset 6). Furthermore, 318 when we compared cortical layer marker expression in iNSCs and pNSCs, 319 we found that compared to pNSCs, iNSC cultures cannot form a subset of 320 neuronal lineages especially for layers II, III and IV in our particular 321 culture conditions (Fig. 3E). These findings suggest that our 3D culture 322 system can be used to dissect the properties and neurogenic capacities of 323 different progenitor types in particular culture settings and under certain 324 physical parameters, and may serve as a suitable tool for investigating 325 the physiological differences between induced and primary NSC 326 populations.

327 Since our culture system can form cortical neurons, the development of 328 which relies on the plasticity and neurogenic ability of human NSCs, we 329 hypothesized that we could model disease conditions that lead to impaired 330 NSC plasticity and neurogenic output. In the human brain, $A \beta 42$ 331 aggregation impairs neuronal network formation and neuronal 332 connectivity due to death of existing neurons as well as loss of neurogenic 333 ability and neural stem cell plasticity (Hardy and Selkoe, 2002; Kienlen- 
334 Campard et al., 2002; LaFerla et al., 2007; Selkoe, 2002; Tincer et al., 335 2016). A 342 was shown to negatively affect the plasticity and 336 neurogenesis of NSCs in mouse models of AD (Ermini et al., 2008; 337 Haughey et al., 2002; Heo et al., 2007), and the same effect is one of the 338 principle limitations of current neuro-regenerative approaches to the treatment of $A D$ in humans (Cosacak et al., 2015; Demars et al., 2010;

340 Tincer et al., 2016). However, the mechanisms underlying the impact of 341 A 342 on NSC plasticity are still largely unknown and cannot be elucidated 342 analytically in human brains. We therefore extended our above-described $3433 \mathrm{D}$ cultures by treating primary NSCs with $A \beta 42$, a major hallmark of $A D$ 344 pathology, before embedding them in the biohybrid hydrogels (Figure 4A). 345 We used an $A \beta 42$ form that we previously found to be causing 346 pathological outcomes in vertebrate brains (Bhattarai et al., 2016; 347 Bhattarai et al., 2017b). In control gels, the neurons formed highly 348 connected networks (Fig. 4B) that were disrupted upon $A \beta 42$ treatment 349 (Fig. 4C). We developed an algorithm to trace the connected neuronal 350 paths as skeletonized arbors and quantified the extent of the neuronal 351 connections, and observed that neuronal networks reduce significantly 352 after Amyloid toxicity (Fig. 4D-F). Similar to human brains, A $\beta 42$ resulted 353 in dystrophic axons (Fig. 4G-G"; Supplementary Video 5), impaired ECM 354 composition and stiffness (Figure $4 \mathrm{H}-\mathrm{J}$ ), Tau hyperphosphorylation (Figure $3554 \mathrm{~K}-\mathrm{N}$, Supplementary Movie 5), neurofibrillary tangle formation as 356 observed by Gallyas silver impregnation (Fig. 4m, Suppl. Fig. 3) and 357 Thioflavin S staining (Fig. 4N), microtubule disassembly (Figure 40), and 358 amyloid aggregation and autophagy (Figure 4P,Q).

359 Amyloid toxicity not only impairs neurogenesis and neuronal survival but 360 also reduces synaptic plasticity (Selkoe, 2002). Amyloid load prevents the 361 formation of new synapses, and newly added cells cannot integrate into 362 the circuitry, rendering exogenous stem cell therapy inefficient (Lilja et 363 al., 2015; Tong et al., 2015). We developed a transplantation paradigm 364 with cultured gels to test whether our 3D culture model could be used to 365 address questions regarding the neurogenic potential and capacity of 366 transplanted cells to integrate into existing networks (Fig. 4R). We labeled 367 all pNSCs with a nuclear stain, and injected them into another hydrogel 
368 that had been pre-cultured with embedded pNSCs for 1 week (Fig. 4R). At

3691 week after transplantation, the injected cells formed neurons with 370 arbors (red nuclei, Fig. 4R; Supplementary Video 7) and connected to pre371 existing cells in the control gels (Fig. $4 R, R^{\prime}$ ). In contrast, cells injected into 372 A 342 -containing gels did not acquire an arborized morphology or connect 373 to the existing cells that were also not arborized (Fig. 4S, S'; 374 Supplementary Video 8). Combined with the findings that A $\beta 42$ impairs 375 the synaptic connections overall (Fig. 4T), these results suggest that 3D 376 cultures can be used for analyzing how synaptic connections can be 377 regenerated and how new neurons can be forced to integrate into the 378 existing circuitry upon $\mathrm{A} \beta 42$ toxicity.

379 A 342 reduces the NSC plasticity and neurogenic capacity in human brains, 380 and stem cell-based regenerative therapies would require therapeutic 381 activation of NSCs (Tincer et al., 2016). To investigate whether $A \beta 42$ 382 reduced NSC plasticity in our 3D cultures, we determined the proliferative 383 capacity and prevalence of pNSCs after $\mathrm{A} \beta 42$ by BrdU/EdU treatment and 384 immunohistochemical stainings for NSC markers SOX2 and GFAP (Fig. 4U$385 \mathrm{X})$. We treated the cultures with BrdU at 1 week of development and with 386 EdU at 2 weeks of development, and analyzed the presence of BrdU and 387 EdU positive cells at 3 weeks of cultures where double-positive cells would 388 indicate constitutively proliferating stem cells (Fig. $4 \mathrm{U}, \mathrm{V}$ ). We found that 389 A 342 reduced BrdU-EdU incorporation and the number of constitutively 390 proliferating cells (Fig. 4Y) as well as reducing numbers of GFAP and 391 SOX2-positive NSCs (Fig. 4Y), indicating that our 3D culture system can 392 be used for modeling A 342 -induced impairment of NSC plasticity. Thus, 393 our 3D cultures of NSCs can also successfully serve as a novel in vitro 394 sporadic AD model of A 442 toxicity on human NSC plasticity and 395 neurogenesis (Figure 4Z). Importantly, we found that all those effects of 396 A 442 observed in $3 D$ cultures are specific because scrambled $A \beta 42$ or 397 other $A \beta$ species such as $A \beta 38$ do not show any phenotypes above (Suppl. 398 Figure 4).

399 To determine if $A \beta 42$ would affect the neuronal network formation and 400 NSC plasticity in iPSC-derived cultures, we treated the iNSCs with A 442 , 401 encapsulated in 3D gels. To determine the neuronal network formation, 
402 NSC prevalence, and proliferative capacity, we performed 403 immmunostainings for GFAP and TUBB3 (Fig. 5A,B), SOX2 and GFAP (Fig. 404 5C,D), and GFAP and BrdU (Fig. 5E,F) together with $A \beta 42$ detection. 405 Compared to controls, $A \beta 42$ reduces the total number of newborn cells 406 and NSCs (GFAP/SOX2-positive) (Fig. 5G), average number of networks 407 (Fig. 5H), average number of branches per network (Fig. 5I), average 408 branch length per network (Fig. 5J), length of longest connected path 409 (Fig. 5K), and maximum branch length (Fig. 5L) in cultures started with 410 iNSCs. These results show that $A \beta 42$ impairs the plasticity, neurogenic 411 ability and network forming capacity of primary and human NSC cultures 412 in our 3D hydrogel matrix, and our system can also be used for iPSC413 derived cell types.

414 To determine the gene expression changes exerted by $A \beta 42$ in 3D 415 cultures, we performed whole transcriptome sequencing on control and 416 Aß42-treated cultures initiated with human iNSCs (Fig. 5M, Suppl. Dataset 417 7) and pNSCs (Fig. 5N, Suppl. Dataset 8). Cellular component analyses 418 and KEGG pathway enrichment analyses in iNSC-derived (Fig. 50, Suppl. 419 Dataset 9, 10) and primary (Fig. 5P, Suppl. Dataset 11, 12) cultures 420 showed that divergent pathways are affected by $A \beta 42$ in 3D cultures. 421 Hierarchical clustering (Fig. 5Q) and multivariance analyses (Fig. 5R) 422 indicated that primary and induced NSC cultures have their own molecular 423 signatures of gene expression, which are affected by $A \beta 42$. By plotting a 424 heat map of gene expression changes, we also found that several cortical 425 marker genes are differentially expressed after Aß42 in induced and 426 primary human NSC-based 3D cultures (Fig. 5S), which is suggestive of 427 the alterations in the cortical neuronal subtypes (for instance, POU3F2, 428 CRYM, and FOXO1). Our results indicate that although $A \beta 42$ causes 429 impaired neural stem cell plasticity, neurogenesis and network formation 430 in both iNSC- and pNSC-derived cultures (Fig. 4, Fig. 5), the molecular 431 programs it alters in these cultures do differ (Fig. 50, P, S). This finding is 432 consistent with previous documentations that primary and iPSC-derived 433 neural stem cells have profound physiological differences that might affect 434 subsequent global gene expression, neuronal maturation capacity, and 435 resilience to disease conditions (Kim et al., 2011; Kim et al., 2010; 
436 Verpelli et al., 2013; Xia et al., 2016). Therefore, our results suggest that

437 starPEG-Heparin 3D culture system can also be used to dissect the effects 438 of $A \beta 42$ on different stem cell and neuronal populations. Furthermore, our $4393 \mathrm{D}$ cultures may help refining the overall toxicity of $A \beta 42$ to distinct 440 physiological states and cellular characteristics of experimental cellular 441 systems. Since our cultures do not contain inflammatory cells, this system 442 will also help to investigate the direct roles of $A \beta 42$ on stem cells and 443 neurons in a reductionist and dissective manner.

\section{Discussion}

445 Neurodegenerative diseases such as AD present with a perplexing set of 446 impairments, including neuronal death, synaptic degeneration, and the 447 inability of stem cells to produce neurons to replace lost neurons (Kienlen448 Campard et al., 2002; LaFerla et al., 2007; Lindvall and Kokaia, 2006; 449 Selkoe, 2002, 2003). Thus, designing effective regenerative therapies for 450 patients with $A D$ requires assay systems that address the parameters of 451 neurodegenerative pathology individually and in combination in a 452 reductionist way using human cells. Rodent models of neurodegeneration 453 cannot recapitulate various aspects of human pathology (Gotz and Ittner, 454 2008; LaFerla and Green, 2012). Thus, we used primary and iPSC-derived 455 human neural stem cells (iNSCs) in 3D cultures to better reflect 456 neurodevelopmental paradigms and neurodegenerative processes in a 457 reductionist manner. 3D cultures using a well-defined biohybrid hydrogel 458 system based on star-shaped PEG and heparin allowed the generation of 459 extensive neuronal networks that could be used to address neurogenesis 460 and connectivity-based questions.

461 Existing 3D culture systems, including Matrigel $^{\mathrm{TM}}$-based cultures, are 462 chemically undefined and heterogeneous in composition and cannot be 463 modified for various parameters, such as stiffness, scaffold composition or 464 bioresponsiveness (Ravi et al., 2015). Thus, the interpretation of often 465 quite variable results is difficult, and it is rarely possible to dissect the 466 influences of different exogenous and paracrine signals on cellular 467 development in an isolated and controllable experimental setup. Our 468 biohybrid hydrogel system based on heparin and star-shaped PEG 469 provides valuable advantages by enabling the independent and ongoing 
470 adjustment of biophysical and biomolecular matrix signals. Indeed, we 471 determined that starPEG-Heparin gels allow faster and more elaborate 472 network formation compared to Matrigel upon identical culture conditions 473 (Suppl. Fig 2). Moreover, the method we use to generate the gels does 474 not cause cell death or DNA mutation that occurs in other matrices 475 through the formation of free radicals upon polymer network formation.

476 Various 3D systems, including organoids, cannot form reproducibly sized 477 or formed structures (Fatehullah et al., 2016). However, MMP-sensitive, 478 starPEG-heparin-based 3D cultures can be customized for all of these 479 parameters, providing better-defined conditions. Compared to previous 480 reports modeling $A D$ in 3D cultures using Matrigel (Choi et al., 2014; 481 Smith et al., 2015), despite differences in the initial cell source, starPEG482 heparin 3D gels enabled significantly faster development of neuronal 483 networks that offer various advantages such as high-throughput screening 484 approaches that are suitable for drug discovery.

485 The composition and architecture of the ECM is an integral parameter 486 governing stem cell activity and tissue modeling. However, due to the 487 complex interplay between multiple ECM-derived signals and their 488 pleiotropic effects, in vivo assays pose a challenge to identifying the roles 489 of exogenous cues in tissue patterning. Previously described methods for 490 the formation of three-dimensional neuronal networks (Choi et al., 2014; 491 Fatehullah et al., 2016; Tang-Schomer et al., 2014; Zhang et al., 2014) 492 lack a defined composition and the ability to tune the artificial 493 extracellular matrix. Our novel 3D matrix platform would be advantageous 494 to dissect the roles of matrix properties in stem cell activity and 495 differentiation, because cells can dynamically interact with the scaffold to 496 generate their "own" cell-secreted ECM. Additionally, our hydrogels can be 497 covalently functionalized with different matrix-derived peptides or could 498 be used for the effective administration of GAG-affine soluble signal 499 molecules. By doing so, the effects of exogenous cues could be 500 individually tested on human neural stem/progenitor cell proliferation and 501 neuronal network formation. Similarly, cells in 3D cultures can be 502 transfected with plasmids for misexpression studies, a powerful tool for 503 rapid investigation of gene function. 
504 Our new 3D culture platform provides a novel and improved model to 505 study the plasticity of human neural stem cells and disease states in real 506 time. The application of customized $A \beta 42$ peptides for optimized cellular 507 uptake within the hydrogel-based 3D cultures indicates that our advanced 508 culture method can recapitulate the major pathophysiology of human 509 A 342 toxicity. Additionally, our gels can be used to experimentally 510 investigate how new cells are incorporated into diseased brains, and how 511 different sources of neural stem cells would molecularly affect the 512 outcomes of $A \beta 42$ toxicity (e.g.: primary versus induced neural stem 513 cells). Individual differences between cell types and their response to 514 disease stimuli can also be measured in a tissue-mimetic composition 515 using our gel system. In overall, starPEG-heparin-hydrogel-based 3D 516 human neural stem cell culture is a novel and comprehensive method for 517 analyzing various stages of neural development and disease of the human 518 cortex, from stem cell proliferation to neurogenesis and from neuronal 519 maturation to integration into the circuitry, in a highly defined and 520 controllable method in an in vitro environment. Our system can be 521 expanded for examining embryonic stem cells, organoids or adult-derived 522 cortical cells from humans, and analyze their stem cell properties or 523 neurogenic capacity in a comparative manner to complement previous 524 studies (Choi et al., 2014; Koutsopoulos and Zhang, 2013; Zhang et al., 525 2014). Beyond that, our established 3D hydrogel cultures can be expected 526 to enable personalized medicine approaches targeting brain diseases or 527 drug efficacy tests.

529 Author contributions: C.P. and C.K. conceived and designed the 530 experiments. M.I.C. analyzed the next generation sequencing data and 531 performed the bioinformatics analyses. L.B., U.F., and C.W. provided the 532 gel materials, J.F. performed AFM studies. C.P. and H.C. performed cell 533 cultures, imaging and quantifications. P.B., H.H. and V.M helped the cell 534 cultures. C.P. optimized the culture conditions for iPSCs and Matrigel. 535 A.K.T. and Y.Z. provided the Amyloid peptides. X.C., S.H. and C.L.A. 536 performed whole-cell patch clamping. C.K. wrote the manuscript, C.K., 537 C.P., U.F., and C.W. revised the manuscript. 


\section{Acknowledgements}

This work was supported by DZNE and Helmholtz Association (VH-NG1021, C.K.), DFG (KI1524/6, C.K.), (AN797/4-1, C.L.A.) and (CRC TR 67, CRC SFB 655, FOR/EXC999, C.W.); and BMBF (PRECIMATRIX-FKZ03XP0083-310117, C.W.). We wish to thank M. Wagner for electrophysiology, R. Szech for image rendering, and T. Kurth for electron microscopy.

\section{References}

Agrawal, S.M., Lau, L., and Yong, V.W. (2008). MMPs in the central nervous system: where the good guys go bad. Semin Cell Dev Biol 19, 42-51.

Alexa, A., Rahnenfuhrer, J., and Lengauer, T. (2006). Improved scoring of functional groups from gene expression data by decorrelating $\mathrm{GO}$ graph structure. Bioinformatics 22, 1600-1607.

Alvarez-Buylla, A., Seri, B., and Doetsch, F. (2002). Identification of neural stem cells in the adult vertebrate brain. Brain Res Bull 57, 751-758. Bhattarai, P., Thomas, A.K., Cosacak, M.I., Papadimitriou, C., Mashkaryan, V., Zhang, Y., and Kizil, C. (2017a). Modeling Amyloid- $\beta 42$ Toxicity and Neurodegeneration in Adult Zebrafish Brain. Journal of Visualized Experiments 128. Bhattarai, P., Thomas, A.K., Papadimitriou, C., Cosacak, M.I., Mashkaryan, V., Froc, C., Kurth, T., Dahl, A., Zhang, Y., and Kizil, C. (2016). IL4/STAT6 signaling activates neural stem cell proliferation and neurogenesis upon Amyloid- $\beta 42$ aggregation in adult zebrafish brain. Cell Reports 17, 941-948.

Bhattarai, P., Thomas, A.K., Zhang, Y., and Kizil, C. (2017b). The effects of aging on Amyloid- $\beta 42$-induced neurodegeneration and regeneration in adult zebrafish brain. Neurogenesis.

Bray, L.J., Binner, M., Holzheu, A., Friedrichs, J., Freudenberg, U., Hutmacher, D.W., and Werner, C. (2015). Multi-parametric hydrogels support 3D in vitro bioengineered microenvironment models of tumour angiogenesis. Biomaterials 53, 609-620.

Capila, I., and Linhardt, R.J. (2002). Heparin-protein interactions. Angew Chem Int Ed Engl 41, 391-412.

Capilla, I., and Linhardt, R.J. (2002). Heparin-protein interactions,. Angew Chem Int Ed Engl 41, 391-412.

Chen, T.W., Wardill, T.J., Sun, Y., Pulver, S.R., Renninger, S.L., Baohan, A., Schreiter, E.R., Kerr, R.A., Orger, M.B., Jayaraman, V., et al. (2013). Ultrasensitive fluorescent proteins for imaging neuronal activity. Nature 499, 295-300.

Choi, S.H., Kim, Y.H., Hebisch, M., Sliwinski, C., Lee, S., D'Avanzo, C., Chen, H., Hooli, B., Asselin, C., Muffat, J., et al. (2014). A three-dimensional human neural cell culture model of Alzheimer's disease. Nature 515, 274-278.

Chwalek, K., Bray, L.J., and Werner, C. (2014). Tissue-engineered 3D tumor angiogenesis models: Potential technologies for anti-cancer drug discovery. Adv Drug Deliv Rev.

Chwalek, K., Levental, K.R., Tsurkan, M.V., Zieris, A., Freudenberg, U., and Werner, C. (2011). Two-tier hydrogel degradation to boost endothelial cell morphogenesis. Biomaterials 32, 9649-9657. 
Cosacak, M.I., Papadimitriou, C., and Kizil, C. (2015). Regeneration, Plasticity, and Induced Molecular Programs in Adult Zebrafish Brain. Biomed Res Int 2015:769763. Demars, M., Hu, Y.S., Gadadhar, A., and Lazarov, O. (2010). Impaired neurogenesis is an early event in the etiology of familial Alzheimer's disease in transgenic mice. $\mathrm{J}$ Neurosci Res 88, 2103-2117.

Discher, D.E., Mooney, D.J., and Zandstra, P.W. (2009). Growth factors, matrices, and forces combine and control stem cells. Science 324, 1673-1677.

Ermini, F.V., Grathwohl, S., Radde, R., Yamaguchi, M., Staufenbiel, M., Palmer, T.D., and Jucker, M. (2008). Neurogenesis and alterations of neural stem cells in mouse models of cerebral amyloidosis. Am J Pathol 172, 1520-1528.

Esler, W.P., and Wolfe, M.S. (2001). A portrait of Alzheimer secretases--new features and familiar faces. Science 293, 1449-1454.

Fabig, G., Kretschmar, S., Weiche, S., Eberle, D., Ader, M., and Kurth, T. (2012). Labeling of ultrathin resin sections for correlative light and electron microscopy. Methods Cell Biol 111, 75-93.

Falcon, S., and Gentleman, R. (2007). Using GOstats to test gene lists for GO term association. Bioinformatics 23, 257-258.

Fatehullah, A., Tan, S.H., and Barker, N. (2016). Organoids as an in vitro model of human development and disease. Nat Cell Biol 18, 246-254.

Freudenberg, U., Sommer, J.U., Levental, K.R., Welzel, P.B., Zieris, A., Chwalek, K., Schneider, K., Prokoph, S., Prewitz, M., Dockhorn, R., et al. (2012). Using Mean Field Theory to Guide Biofunctional Materials Design. Adv Funct Mater 22, 13911398.

Gage, F.H. (2000). Mammalian neural stem cells. Science 287, 1433-1438.

Gage, F.H., and Temple, S. (2013). Neural stem cells: generating and regenerating the brain. Neuron 80, 588-601.

Garg, H.G., Linhardt, R.J., and Hales, C.A. (2011). Chemistry and Biology of Heparin and Heparan Sulfate (Elsevier).

Gotz, J., and Ittner, L.M. (2008). Animal models of Alzheimer's disease and frontotemporal dementia. Nat Rev Neurosci 9, 532-544.

Haass, C., and Selkoe, D.J. (2007). Soluble protein oligomers in neurodegeneration: lessons from the Alzheimer's amyloid beta-peptide. Nat Rev Mol Cell Biol 8, 101112.

Hardy, J., and Selkoe, D.J. (2002). The amyloid hypothesis of Alzheimer's disease: progress and problems on the road to therapeutics. Science 297, 353-356.

Haughey, N.J., Liu, D., Nath, a., Borchard, a.C., and Mattson, M.P. (2002).

Disruption of neurogenesis in the subventricular zone of adult mice, and in human cortical neuronal precursor cells in culture, by amyloid beta-peptide: implications for the pathogenesis of Alzheimer's disease. Neuromolecular Med 1, 125-135.

Haycock, J.W. (2011). 3D cell culture: a review of current approaches and techniques. Methods Mol Biol 695, 1-15.

Heneka, M.T., Carson, M.J., El Khoury, J., Landreth, G.E., Brosseron, F., Feinstein, D.L., Jacobs, A.H., Wyss-Coray, T., Vitorica, J., Ransohoff, R.M., et al. (2015). Neuroinflammation in Alzheimer's disease. The Lancet Neurology 14, 388-405. Heo, C., Chang, K.-A., Choi, H.S., Kim, H.-S., Kim, S., Liew, H., Kim, J.a., Yu, E., Ma, J., and Suh, Y.-H. (2007). Effects of the monomeric, oligomeric, and fibrillar A $\beta 42$ peptides on the proliferation and differentiation of adult neural stem cells from subventricular zone. Journal of Neurochemistry 102, 493-500.

Justice, B.A., Badr, N.A., and Felder, R.A. (2009). 3D cell culture opens new dimensions in cell-based assays. Drug Discov Today 14, 102-107. 
Kienlen-Campard, P., Miolet, S., Tasiaux, B., and Octave, J.N. (2002). Intracellular amyloid-beta 1-42, but not extracellular soluble amyloid-beta peptides, induces neuronal apoptosis. J Biol Chem 277, 15666-15670.

Kim, J., Efe, J.A., Zhu, S., Talantova, M., Yuan, X., Wang, S., Lipton, S.A., Zhang, K., and Ding, S. (2011). Direct reprogramming of mouse fibroblasts to neural progenitors. Proc Natl Acad Sci U S A 108, 7838-7843.

Kim, K., Doi, A., Wen, B., Ng, K., Zhao, R., Cahan, P., Kim, J., Aryee, M.J., Ji, H., Ehrlich, L.I., et al. (2010). Epigenetic memory in induced pluripotent stem cells. Nature 467, 285-290.

Kizil, C., Iltzsche, A., Kuriakose, A., Bhattarai, P., Zhang, Y., and Brand, M. (2015). Efficient cargo delivery using a short cell-penetrating peptide in vertebrate brains. PLoS One 10, e0124073.

Koutsopoulos, S., and Zhang, S. (2013). Long-term three-dimensional neural tissue cultures in functionalized self-assembling peptide hydrogels, matrigel and collagen I. Acta Biomater 9, 5162-5169.

LaFerla, F.M., and Green, K.N. (2012). Animal models of Alzheimer disease. Cold Spring Harbor perspectives in medicine 2.

LaFerla, F.M., Green, K.N., and Oddo, S. (2007). Intracellular amyloid-beta in Alzheimer's disease. Nat Rev Neurosci 8, 499-509.

Lau, L.W., Cua, R., Keough, M.B., Haylock-Jacobs, S., and Yong, V.W. (2013). Pathophysiology of the brain extracellular matrix: a new target for remyelination. Nat Rev Neurosci 14, 722-729.

Liao, Y., Smyth, G.K., and Shi, W. (2013). The Subread aligner: fast, accurate and scalable read mapping by seed-and-vote. Nucleic Acids Res 41, e108.

Liao, Y., Smyth, G.K., and Shi, W. (2014). featureCounts: an efficient general purpose program for assigning sequence reads to genomic features. Bioinformatics 30, 923-930. Lilja, A.M., Malmsten, L., Röjdner, J., Voytenko, L., Verkhratsky, A., Ögren, S.O., Nordberg, A., and Marutle, A. (2015). Neural Stem Cell Transplant-Induced Effect on Neurogenesis and Cognition in Alzheimer Tg2576 Mice Is Inhibited by Concomitant Treatment with Amyloid-Lowering or Cholinergic $\alpha 7$ Nicotinic Receptor Drugs. Neural Plast 2015, 370432. Lindvall, O., and Kokaia, Z. (2006). Stem cells for the treatment of neurological disorders. Nature 441, 1094-1096.

Love, M.I., Huber, W., and Anders, S. (2014). Moderated estimation of fold change and dispersion for RNA-seq data with DESeq2. Genome biology 15, 550. Luo, W., and Brouwer, C. (2013). Pathview: an R/Bioconductor package for pathwaybased data integration and visualization. Bioinformatics 29, 1830-1831. Lutolf, M.P., Gilbert, P.M., and Blau, H.M. (2009). Designing materials to direct stem-cell fate. Nature 462, 433-441.

Maitz, M.F., Freudenberg, U., Tsurkan, M.V., Fischer, M., Beyrich, T., and Werner, C. (2013). Bio-responsive polymer hydrogels homeostatically regulate blood coagulation. Nat Commun 4, 2168.

Molyneaux, B.J., Arlotta, P., Menezes, J.R., and Macklis, J.D. (2007). Neuronal subtype specification in the cerebral cortex. Nat Rev Neurosci 8, 427-437. Nalbantoglu, J., Tirado-Santiago, G., Lahsaini, A., Poirier, J., Goncalves, O., Verge, G., Momoli, F., Welner, S.A., Massicotte, G., Julien, J.P., et al. (1997). Impaired learning and LTP in mice expressing the carboxy terminus of the Alzheimer amyloid precursor protein. Nature 387, 500-505. 
682

683

684

685

686

687

688

689

690

691

692

693

694

695

696

697

698

699

700

701

702

703

704

705

706

707

708

709

710

711

712

713

714

715

716

717

718

719

720

721

722

723

724

725

726

727

728

729

730

Ravi, M., Paramesh, V., Kaviya, S.R., Anuradha, E., and Solomon, F.D. (2015). 3D

cell culture systems: advantages and applications. J Cell Physiol 230, 16-26.

Selkoe, D.J. (2002). Alzheimer's disease is a synaptic failure. Science 298, 789-791.

Selkoe, D.J. (2003). Folding proteins in fatal ways. Nature 426, 900-904.

Smith, I., Silveirinha, V., Stein, J.L., de la Torre-Ubieta, L., Farrimond, J.A.,

Williamson, E.M., and Whalley, B.J. (2015). Human neural stem cell-derived cultures in three-dimensional substrates form spontaneously functional neuronal networks. $\mathrm{J}$

Tissue Eng Regen Med.

Tang-Schomer, M.D., White, J.D., Tien, L.W., Schmitt, L.I., Valentin, T.M.,

Graziano, D.J., Hopkins, A.M., Omenetto, F.G., Haydon, P.G., and Kaplan, D.L.

(2014). Bioengineered functional brain-like cortical tissue. Proc Natl Acad Sci U S A $111,13811-13816$.

Tincer, G., Mashkaryan, V., Bhattarai, P., and Kizil, C. (2016). Neural

stem/progenitor cells in Alzheimer's disease. Yale J Biol Med 89, 23-35.

Tong, L.M., Fong, H., and Huang, Y. (2015). Stem cell therapy for Alzheimer's

disease and related disorders: current status and future perspectives. Experimental \& molecular medicine 47, e151.

Tsurkan, M.V., Chwalek, K., Levental, K.R., Freudenberg, U., and Werner, C. (2011). Modular StarPEG-Heparin Gels with Bifunctional Peptide Linkers. Macromol Rapid Commun 31, 1529-1533.

Tsurkan, M.V., Chwalek, K., Prokoph, S., Zieris, A., Levental, K.R., Freudenberg, U., and Werner, C. (2013). Defined polymer-peptide conjugates to form cell-instructive starPEG-heparin matrices in situ. Adv Mater 25, 2606-2610.

Tsurkan, M.V., Levental, K.R., Freudenberg, U., and Werner, C. (2010).

Enzymatically degradable heparin-polyethylene glycol gels with controlled

mechanical properties. Chem Commun (Camb) 46, 1141-1143.

Venable, J.H., and Coggeshall, R. (1965). A Simplified Lead Citrate Stain for Use in Electron Microscopy. J Cell Biol 25, 407-408.

Verpelli, C., Carlessi, L., Bechi, G., Fusar Poli, E., Orellana, D., Heise, C.,

Franceschetti, S., Mantegazza, R., Mantegazza, M., Delia, D., et al. (2013).

Comparative neuronal differentiation of self-renewing neural progenitor cell lines obtained from human induced pluripotent stem cells. Front Cell Neurosci 7, 175.

Wieduwild, R., Tsurkan, M., Chwalek, K., Murawala, P., Nowak, M., Freudenberg, U., Neinhuis, C., Werner, C., and Zhang, Y. (2013). Minimal peptide motif for noncovalent peptide-heparin hydrogels. J Am Chem Soc 135, 2919-2922.

Wu, T.D., Reeder, J., Lawrence, M., Becker, G., and Brauer, M.J. (2016). GMAP and GSNAP for Genomic Sequence Alignment: Enhancements to Speed, Accuracy, and Functionality. Methods Mol Biol 1418, 283-334.

Wyss-Coray, T. (2016). Ageing, neurodegeneration and brain rejuvenation. Nature 539, 180-186.

Xia, N., Zhang, P., Fang, F., Wang, Z., Rothstein, M., Angulo, B., Chiang, R., Taylor, J., and Reijo Pera, R.A. (2016). Transcriptional comparison of human induced and primary midbrain dopaminergic neurons. Sci Rep 6, 20270.

Young, M.D., Wakefield, M.J., Smyth, G.K., and Oshlack, A. (2010). Gene ontology analysis for RNA-seq: accounting for selection bias. Genome biology 11, R14.

Yu, G., Wang, L.G., Han, Y., and He, Q.Y. (2012). clusterProfiler: an R package for comparing biological themes among gene clusters. OMICS 16, 284-287.

Zhang, D., Pekkanen-Mattila, M., Shahsavani, M., Falk, A., Teixeira, A.I., and Herland, A. (2014). A 3D Alzheimer's disease culture model and the induction of 
731 P21-activated kinase mediated sensing in iPSC derived neurons. Biomaterials 35, 732 1420-1428.

733 Zhang, Y., Fussel, S., Reimer, U., Schutkowski, M., and Fischer, G. (2002).

734 Substrate-based design of reversible Pin1 inhibitors. Biochemistry 41, 11868-11877.

735

736 


\section{Main figures and legends}

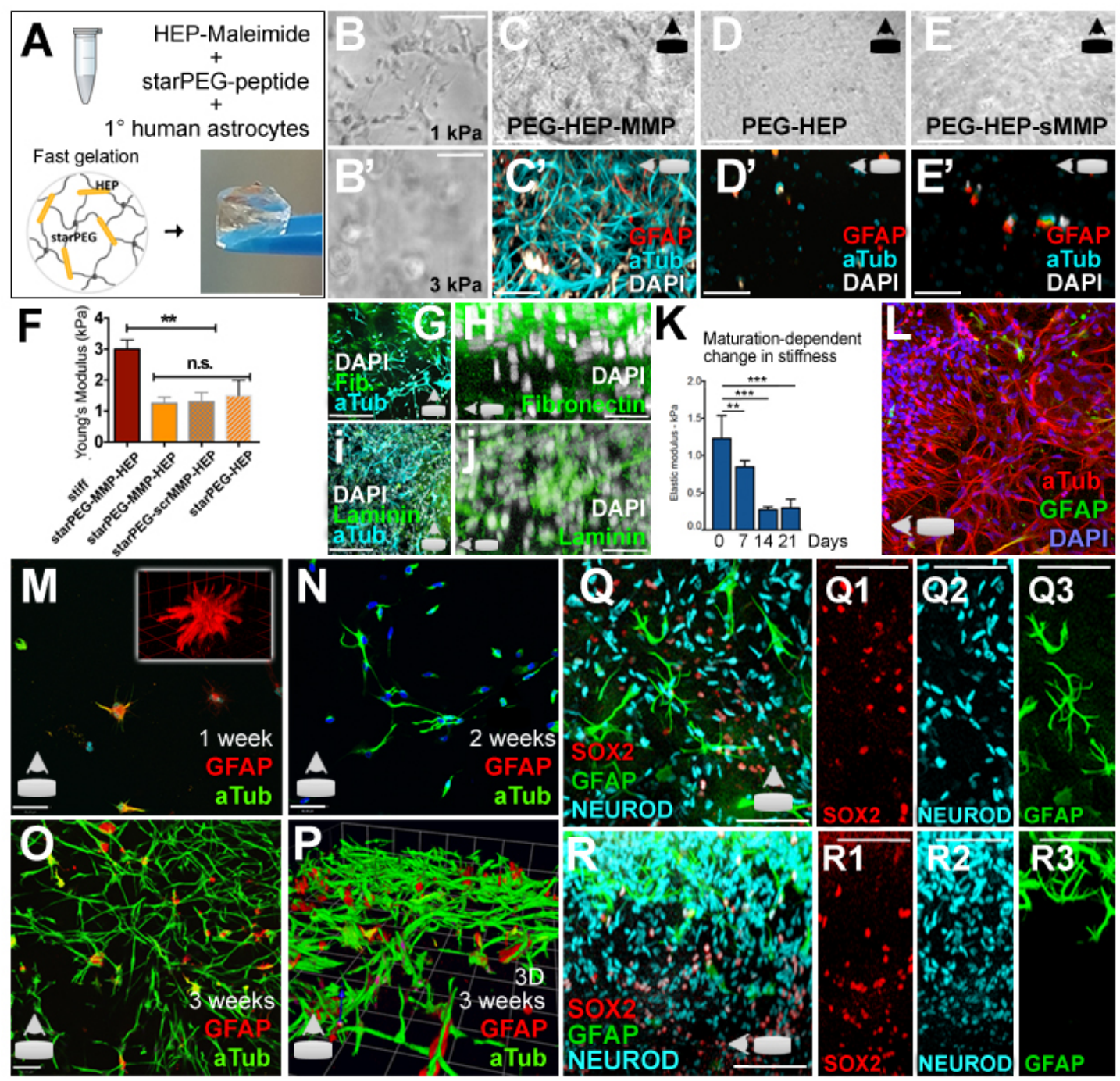

Figure 1

740 Physical properties and dynamic remodeling of 3D starPEG-Heparin

741 hydrogels and tissue-mimetic behavior of primary human neural

742 stem/progenitor cells (NSPCs).

744 (A) Simplified composition and preparation scheme of glycosaminoglycan

745 (GAG)-based minimalist multifunctional hydrogels.

746 (B, B') Bright field gel image (BFI) at 1-kPa (B) and 3-kPa (B') stiffness.

747 (C-E') BFI $(C, D, E)$ and immunostaining for acetylated-tubulin (aTub) and

748 GFAP $\left(C^{\prime}, D^{\prime}, E^{\prime}\right)$ in a 3-week-old gel at $1 \mathrm{kPa}$ stiffness with $\left(\mathrm{C}, \mathrm{C}^{\prime}\right)$, without

$749\left(D, D^{\prime}\right)$ and with scrambled MMP cleavage sites $\left(E, E^{\prime}\right)$.

750 (F) Rheological measurements of various gel types.

751 (G) Acetylated-tubulin and fibronectin. 
752 (H) Fibronectin.

753 (I) Acetylated-tubulin and Iaminin.

754 (J) Laminin.

755 (K) Atomic force microscopy-based quantification of elastic modulus in the 756 development of the neural networks.

757 (L) Maximum projection for TUBB3 and GFAP (X-axis).

758 (M-O) Acetylated-tubulin/GFAP staining over the $z$-axis after $1(\mathrm{M}), 2(\mathrm{~N})$, 759 and $3(0)$ weeks of culture.

760 (P) 3D representation for acetylated-tubulin and GFAP.

761 (Q-R3) Maximum projection images for SOX2, GFAP, and NEUROD1 over 762 the Z-axis (Q-Q3) and X-axis (R-R3). Single fluorescent channels are 763 shown on the right (Q1-Q3, R1-R3).

764 Scale bars: $10 \mu \mathrm{m}$ for $\mathrm{H}, \mathrm{J}$, and $\mathrm{M}-\mathrm{P} ; 50 \mu \mathrm{m}$ for the other figures. Gels: 3 765 weeks of culture. See also Supplementary Movies 1, 2, and 3.
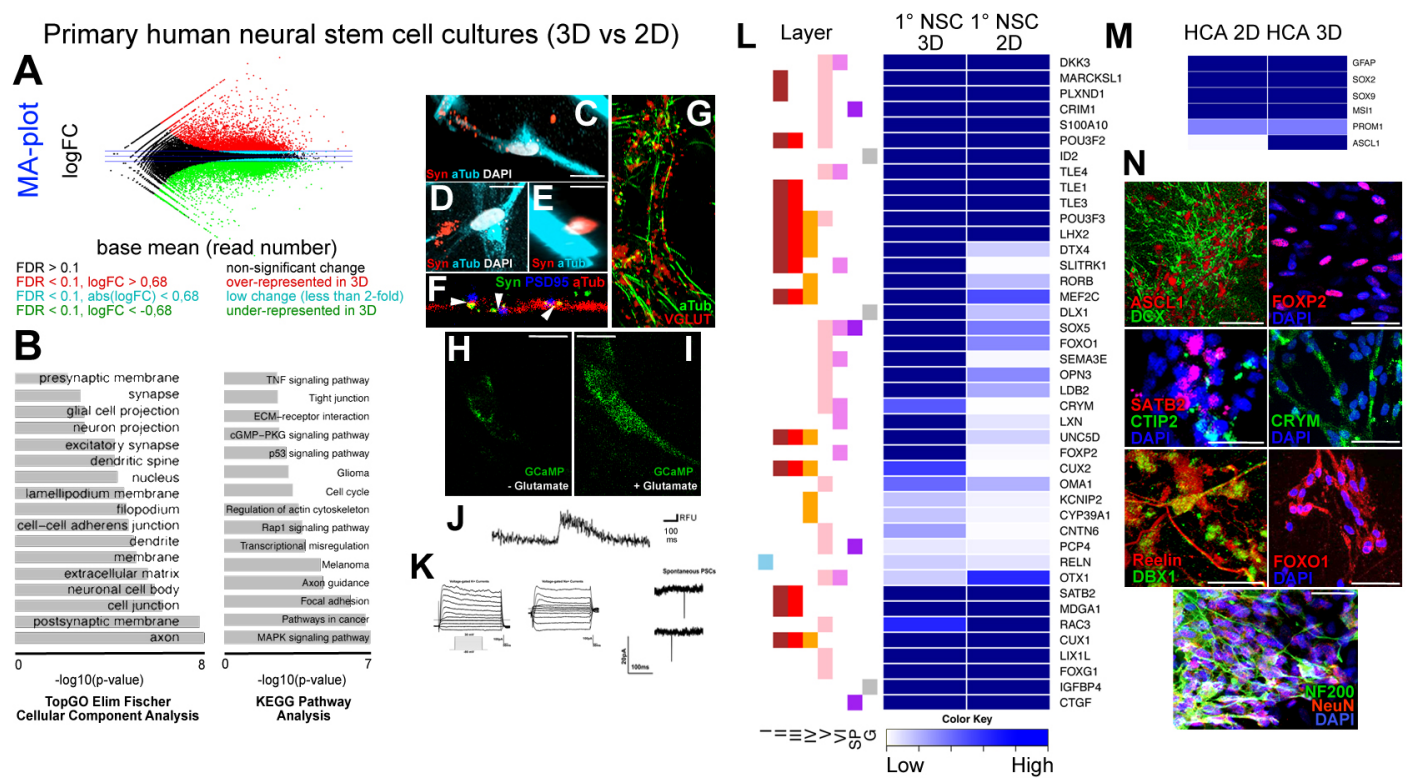

769 Figure 2

770 Comparison of gene expression profiles of primary human cortical 771 astrocytes in 2D and 3D cultures.

773 (A) MA-plot for differentially expressed genes. Red: upregulated in 3D, 774 green: downregulated in 3D. 
775 (B) KEGG pathway analyses pie chart showing significantly enriched 776 molecular pathways.

777 (C-E) Synaptophysin and Acetylated tubulin immunostaining in 3D 778 cultures.

779 (F) Synaptophysin, PSD95 and Acetylated tubulin immunostaining in 3D 780 cultures.

781 (G) VGLUT1 and Acetylated tubulin immunostaining in 3D cultures.

782 (H) GCaMP6 signal without Glutamate treatment.

783 (I) GCaMP6 signal with Glutamate treatment.

784 (J) Fluorescence intensity histogram. Note the peak at the time of 785 Glutamate treatment.

786 (K) Patch clamp recordings for $\mathrm{Na}+$ (left) and $\mathrm{K}+$ (middle) currents. 787 Detection of spontaneous firing of neurons (right).

788 (L) Heat map for expression levels of cortical marker genes for 2D and 3D 789 cultures. Asterisks indicate significant difference between two samples. 790 Genes are denoted with their respective cortical layer expression with 791 color codes (left to the heat map).

792 (M) Heat map for expression levels of neural stem cell markers.

793 (N) Immunostaining for ASCL1 (proneural determinant), DCX (early 794 neuronal marker), SATB2, CTIP2, Reelin (RELN), DBX1, FOXP2, CRYM, 795 FOXO1 (cortical markers), and Neurofilament (NF200) and NeuN (mature 796 neuronal markers).

797 Scale bars: $10 \mu \mathrm{m}$ for C-I; $25 \mu \mathrm{m}$ for N. Gels: 3 weeks of culture. See also 798 Supplementary Datasets 1, 2 and 3. 
802

803

804

805

806

807

808

809

810 (white).

811 (B) Immunostaining for GFAP (red) and TUBB3 (cyan) on 3 weeks of 3D 812 cultures with iPSC-derived human neural stem cells. Insets below the 813 panel are individual fluorescence channels including DAPI (white).

814 (C) MA-plot for differentially expressed genes in primary over iPSC815 derived NSC cultures. Red: over-represented in primary cultures, green: 816 underrepresented in primary cultures compared to iPSC cultures.

817 (D) KEGG pathway analyses pie chart showing significantly enriched 818 molecular pathways in primary cultures compared to iPSC cultures.

819 (E) Heat map for expression levels of cortical marker genes in iPSC and 820 primary cultures. Asterisks indicate significant difference between two 821 samples. Genes are denoted with their respective cortical layer expression 822 with color codes (left to the heat map).

823 Scale bars: $100 \mu \mathrm{m}$. Gels: 3 weeks of culture.

824 See also Supplementary Datasets 4, 5 and 6. 

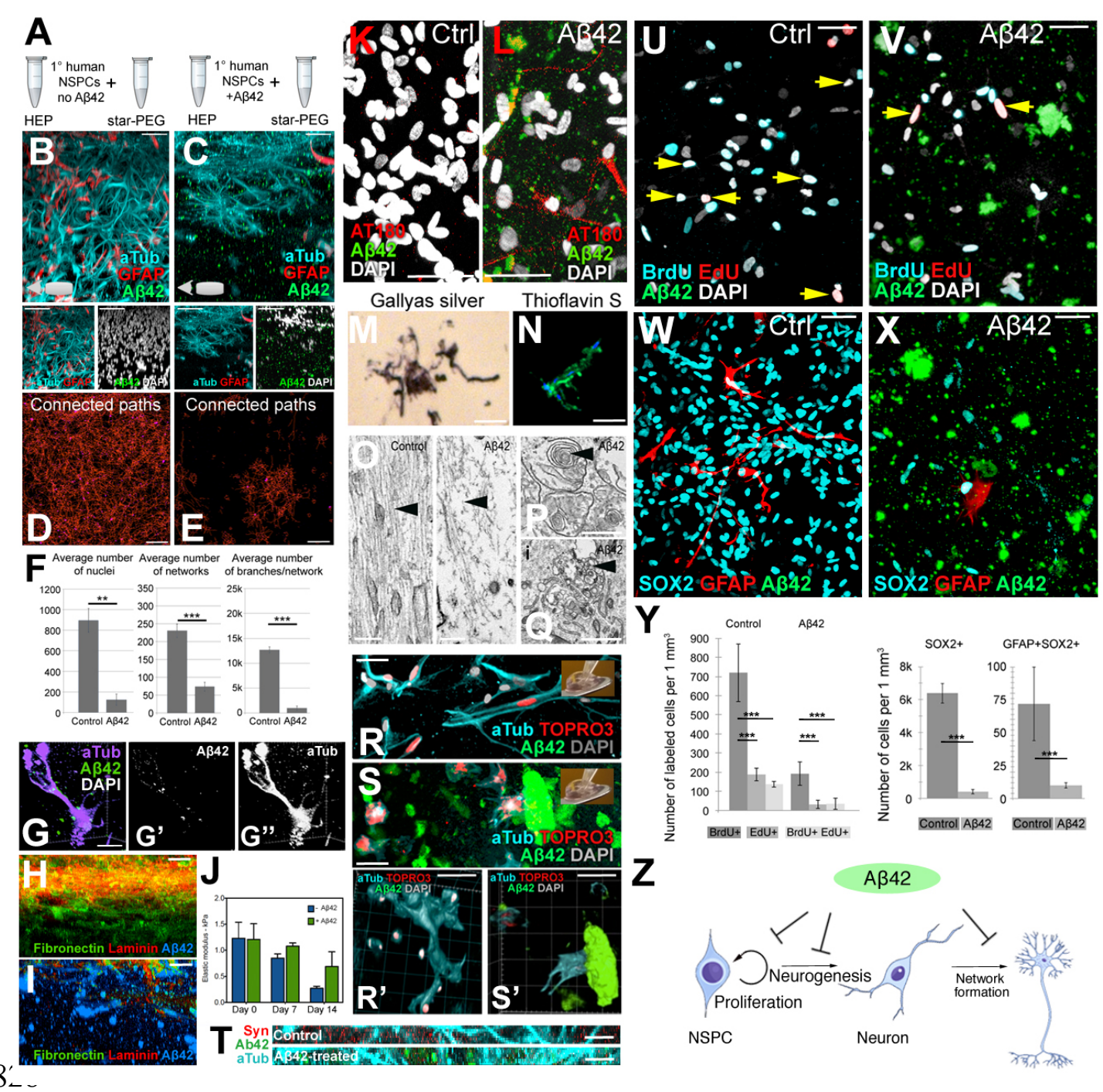

829 Figure 4

$8303 D$ hydrogel cultures of primary human NSCs as an A 342 toxicity model.

832 (A) Simplified gel preparation/A 442 administration scheme.

$833(B, C) X$-axis views for $A \beta 42$, Acetylated-tubulin, GFAP in control (B) and 834 A 442 gels $(C)$. Double stainings under the panels.

$835(D, E)$ Maximum projection of the skeletonized-connected neuronal 836 pathways in control (D) and $A \beta 42$ gels (E).

837 (F) Quantification.

$838\left(\mathrm{G}-\mathrm{G}^{\prime \prime}\right)$ 3D-reconstruction of TUBB3/Aß42 showing dystrophic axons.

$839(\mathrm{H}, \mathrm{I})$ Fibronectin/laminin in control $(\mathrm{H})$ and $A \beta 42$ gels (I).

840 (J) Time-course atomic force microscopy measurements of stiffness.

$841(\mathrm{~K}, \mathrm{~L})$ Phospho-Tau (AT180) in control (K) and $\mathrm{A} \beta 42$ gels (L).

842 (M) Gallyas silver staining. 
843 (N) Thioflavin-S staining in A 342 gels.

844 (O) EM images of microtubules in control (left) and $A \beta 42$ gels (right).

$845(P, Q) A \beta 42$ depositions ( $P)$, and autophagic vacuoles (Q).

846 (R-S') Acetylated-tubulin and $A \beta 42$ in control (R) and $A \beta 42$ gels (S) after

847 transplantation. Transplanted cell nuclei labeled with TOPRO3 (red). 3D848 reconstruction of $R\left(R^{\prime}\right)$ and $S\left(S^{\prime}\right)$.

849 ( $T$ ) Acetylated-tubulin, synaptophysin, $A \beta 42$ in control (upper) and $A \beta 42$ 850 gels (lower).

$851(\mathrm{U}, \mathrm{V})$ BrdU and EdU in control (U) and $A \beta 42$ gels (V). Arrows: double852 positive cells.

$853(W, X)$ SOX2, GFAP, $A \beta 42$ in control $(W)$ and $A \beta 42$ gels $(X)$.

854 (Y) Quantification of U-X.

855 (Z) Schematics for effects of $A \beta 42$.

856 Scale bars: $10 \mu \mathrm{m}$ for $\mathrm{G}, \mathrm{M}, \mathrm{N}, \mathrm{T}, \mathrm{R}$, and $\mathrm{S} ; 200 \mathrm{~nm}$ for $\mathrm{O}-\mathrm{Q} ; 50 \mu \mathrm{m}$ for 857 the other figures. Gels: 3 weeks of culture.

858 See also Supplementary Movies 4-8.

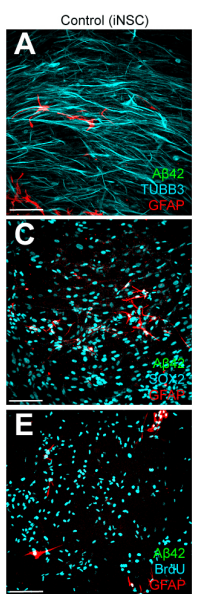

Q

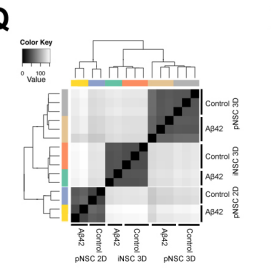

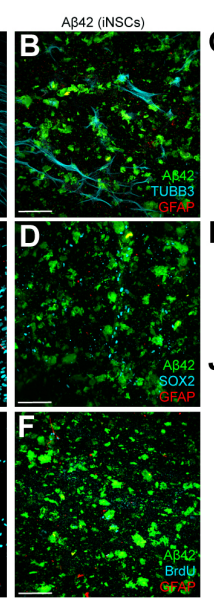

$\mathbf{R}$

\section{$861 \quad$ Figure 5}

862 A 442 toxicity model with iPSC-derived neural stem cells in 3D hydrogels, 863 analysis of transcriptional changes, and comparison to pNSC cultures. 
865 (A) Immunostaining for AB42 (green), TUBB3 (cyan) and GFAP (red) on 866 control iPSC-derived NSC cultures.

867 (B) Immunostaining for A 442 (green), TUBB3 (cyan) and GFAP (red) on 868 A 4 42-treated iPSC-derived NSC cultures.

869 (C) Immunostaining for A 442 (green), SOX2 (cyan) and GFAP (red) on 870 control iPSC-derived NSC cultures.

871 (D) Immunostaining for $A \beta 42$ (green), SOX2 (cyan) and GFAP (red) on 872 A 4 42-treated iPSC-derived NSC cultures.

873 (E) Immunostaining for $A \beta 42$ (green), BrdU (cyan) and GFAP (red) on 874 control iPSC-derived NSC cultures. BrdU is given at 1 week of culture.

875 (F) Immunostaining for AB42 (green), TUBB3 (cyan) and GFAP (red) on 876 A 42 -treated iPSC-derived NSC cultures. BrdU is given at 1 week of 877 culture.

878 (G) Quantification graph for number of cells in control and A 342 -treated 879 cultures.

$880(\mathrm{H}-\mathrm{L})$ Quantification of average number of networks $(\mathrm{H})$, average number 881 of branches per network (I), average branch length per network (J), 882 length of longest connected path (K), and maximum branch length $(L)$.

883 (M) MA-plot for differentially expressed genes in iPSC-derived NSC 884 cultures after $A \beta 42$ treatment. Red: upregulated, green: downregulated.

885 (N) MA-plot for differentially expressed genes in primary NSC cultures 886 after $A \beta 42$ treatment. Red: upregulated, green: downregulated.

887 (O) KEGG pathway analyses pie chart showing significantly enriched 888 molecular pathways in iPSC cultures after A 42 treatment. .

889 (P) KEGG pathway analyses pie chart showing significantly enriched 890 molecular pathways in primary cultures after $A \beta 42$ treatment.

891 (Q) Heat map for changes in expression levels of cortical marker genes in 892 iPSC and primary cultures after A 342 treatment. Genes are denoted with 893 their respective cortical layer expression with color codes (above the heat 894 map).

895 Scale bars: $100 \mu \mathrm{m}$. Gels: 3 weeks of culture.

896 See also Supplementary Datasets 7-12. 


\section{Materials and Methods}

\section{Cell Culture}

901 Astrocytes isolated from the cerebral cortex at gestation week 21 were 902 obtained from ScienCell Research Laboratory (SRL, Catalog Number 1800, 903 Carlsbad, CA, USA) at passage one and delivered as frozen stocks. The 904 cells (from here on primary human neural stem cells, pNSC) are certified 905 to be negative for HIV-1, HBV, HCV, mycoplasma, bacteria, yeast, and 906 fungi. PHCCs were seeded on conventional T75 flasks or 24-well plates and cultured in complete astrocyte medium (AM) composed of Astrocyte medium (SRL, Catalog Number 1801) supplemented with $2 \%$ fetal bovine serum (SRL, Catalog Number 0010), 1\% astrocyte growth supplement

910 (SRL, Catalog Number 1852) and 1\% penicillin/streptomycin solution 911 (SRL, Catalog Number 0503) in an incubator with a 5\% CO2/95\% air 912 atmosphere at $37^{\circ} \mathrm{C}$. Human induced neural stem cells (HIP, BC1 line, 913 Amsbio, catalog number GSC-4311, from here on iNSC) were seeded in 914 Geltrex pre-coated cultureware in complete AM as described in the above 915 paragraph.

\section{Generation of starPEG-Heparin hydrogels and cell encapsulation}

917 StarPEG-heparin hydrogels were generated as previously described (Maitz 918 et al., 2013; Wieduwild et al., 2013) with the following modifications: 919 pNSCs or iNSCs were collected from culture flasks using Accutase 920 (Invitrogen, CA, USA). After centrifugation (271 g for 10 minutes), cells 921 were resuspended in PBS at a concentration of $8 \times 10^{6}$ cells per $\mathrm{ml}$. For 922 each hydrogel, we first resuspended the cells in $5 \mu$ of PBS, then added 5 $923 \mu \mathrm{l}$ Heparin maleimide conjugate solution $(90 \mu \mathrm{g} / \mu \mathrm{l}$ in PBS) and $10 \mu \mathrm{l}$ 924 starPEG-MMP-peptide conjugate solution (Tsurkan et al., 2011) to a final 925 volume of $20 \mu \mathrm{l}$ and a cell density of $2 \times 10^{6} \mathrm{cells} / \mathrm{ml}$. Next, the $20 \mu \mathrm{l}$ 926 droplet was placed on a Parafilm sheet for approximately two minutes 927 until it began to gelate. The gels were placed in 24-well plates, and each 928 well contained $1 \mathrm{ml}$ of astrocyte culture medium (AM) (Supplementary 929 Movie 8). The gels were cultured and incubated in 5\% CO2/95\% air at 37 $930{ }^{\circ} \mathrm{C}$ until the desired time points ( 1 week, 2 weeks, and 3 weeks). 
932 For the generation of Matrigel cultures, we used BD Matrigel (catalog 933 number: 356234). Prior to any cell culture work and use of the Matrigel, 934 pipette tips and Eppendorf tubes has been frozen at $-20{ }^{\circ} \mathrm{C}$ according to 935 manufacturer's instruction following the "thick gel method". The BD 936 Matrigel has been thawed overnight on ice at $4{ }^{\circ} \mathrm{C}$. pNSCs were collected 937 from culture flasks using Accutase (Invitrogen). After centrifugation (271 $938 \mathrm{~g}$ for 10 minutes), pNSCs were re-suspended in BD Matrigel in 939 concentration $2 \times 10^{6}$ cells per $\mathrm{ml}$. Droplets of the cell/Matrigel mix were 940 placed in the bottom of culture and then waiting to solidify at $37^{\circ} \mathrm{C}$. Then, 941 cell medium (SRL, Catalog Number 1801) added and the gels cultured for 9423 weeks (Supplementary Movie 8). Cell medium changed the day after the 943 generation of gels and then every other day.

\section{Synthesis of Amyloid peptides}

945 The peptides were synthesized as previously described (Bhattarai et al., 946 2017a; Bhattarai et al., 2016; Bhattarai et al., 2017b; Kizil et al., 2015; 947 Wieduwild et al., 2013). For peptide synthesis, all of the required 948 chemicals were purchased from IRIS Biotech $\mathrm{GmbH}$ (Marktredwitz, 949 Germany). Acetonitrile (for UPLC/LCMS), dichloromethane (DCM), 950 diethylether, dimethyl sulfoxide (DMSO), formic acid (FA), trifluoroacetic 951 acid (TFA), and triisopropylsilane (TIS) were purchased from MERCK KGaA 952 (Darmstadt, Germany). Acetic anhydride and N-methylmorpholine (NMM) 953 were purchased from Sigma-Aldrich Co. LLC (St. Louis, MO, USA). 954 Dithiothreitol (DTT) was obtained from Prolab VWR International, LCC 955 (Radnor, PA, USA). Acetonitrile (for HPLC) was purchased from TH Geyer 956 (Renningen, Germany). 5(6)-Carboxyfluorescein was purchased from 957 Acros Organics (Fisher Scientific Company LLC). The TentaGel S RAM 958 Fmoc rink amide resin was purchased from RappPolymere $\mathrm{GmbH}$ 959 (Tübingen, Germany). The peptide synthesis columns and syringes with 960 included filters were purchased from Intavis AG (Cologne, Germany). 961 Water was obtained from a Milli-Q water purifier (Milli-Q Advantage A10, 962 EMD Millipore Corporation, Billerica, MA, USA) with a LCPAK0001 Milli-Q 963 filter. The polytetrafluoroethylene (PTFE) filter, polyvinylidene fluoride 964 (PVDF) syringe filter, and filter holder were purchased from Sartorius 965 Stedtim (Aubagne, France). 
966 A 442 peptides were prepared using standard 9-fluorenylmethoxycarbonyl 967 (Fmoc) chemistry with 2-(1H-benzotriazol-1-yl)-1,1,3,3968 tetramethyluronoium hexafluorophosphate (HBTU) activation on an 969 automated solid-phase peptide synthesizer (ResPep SL, 970 Intavis)(Wieduwild et al., 2013; Zhang et al., 2002). Each amino acid was 971 coupled twice at 5 -fold excess followed by capping the non-reacted amino 972 groups with acetic anhydride to achieve high quality synthesis. Upon 973 completion of the peptide synthesis, 5(6)-carboxyfluorescein was coupled 974 to the N-terminus using HBTU as the coupling reagent. The peptide was 975 then cleaved from the resin with TFA/TIS/water/DTT $976(90(\mathrm{v} / \mathrm{v}): 5(\mathrm{v} / \mathrm{v}): 2.5(\mathrm{v} / \mathrm{v}): 2.5(\mathrm{~m} / \mathrm{v}))$ for 2 hours. The product was 977 precipitated and washed with ice-cold diethyl ether.

978 The peptide was dissolved in Milli-Q water, and peptide purification was 979 performed via reverse-phase high-pressure liquid chromatography (HPLC) 980 on a semi-preparative HPLC (Waters) equipped with a semi-preparative 981 column (PolymerX RP-1, $250 \times 10 \mathrm{~mm}$, Phenomenex). The peptide was 982 eluted from the column by applying a gradient of $5 \%$ to $100 \%$ solvent $B$ 983 over $30 \mathrm{~min}$ at $20 \mathrm{ml} / \mathrm{min}$, in which solvent $A$ is $0.1 \%$ TFA in water and 984 solvent $\mathrm{B}$ is $0.1 \%$ TFA and $5 \%$ water in acetonitrile.

985 Purity was confirmed on an analytical reverse phase ultra-high pressure 986 liquid chromatograph (UPLC Aquity with UV Detector) equipped with an 987 analytical C18 column (Acquity UPLC BEH C18, bead size $1.7 \mu \mathrm{m}, 50 \times 2.1$ $988 \mathrm{~mm}$ ) using an isocratic gradient and electrospray ionization mass 989 spectrometry (ESI-MS) (Acquity TQ Detector).

\section{A 342 treatment}

991 The $A \beta 42$ treatment was performed $24 \mathrm{~h}$ post-thaw for a period of $48 \mathrm{~h}$ at $99210 \mu \mathrm{M}$ final concentration for pNSCs and iNSCs in 3D cultures. Amyloid 993 plaques remain in the culture attached to the cells throughout the 994 cultures. The medium was removed and the cells were washed with PBS 995 after 48 h A 442 treatment. Then, the cells were collected using Accutase, 996 counted and centrifuged at $271 \mathrm{~g}$ for $10 \mathrm{~min}$. The cell pellet was 997 resuspended in PBS to obtain at $8 \times 10^{6} \mathrm{cells} / \mathrm{ml}$. This cell suspension was 998 mixed with an equal volume of $6.00 \mathrm{mM}$ heparin maleimide conjugate in 
999 PBS to obtain a $3.00 \mathrm{mM}$ heparin maleimide conjugate-cell suspension

$1000 \mathrm{mix}$ at $4.0 \times 10^{6} \mathrm{cells} / \mathrm{ml}$. $10 \mu \mathrm{l}$ of this mix was combined with $10 \mu \mathrm{l}$ of 10012.25 mM starPEG-MMP-peptide conjugate solution in PBS (Tsurkan et al., 1002 2011), quickly triturated a few times, and the resulting $20 \mu$ l volume was pipetted onto a Parafilm sheet forming a droplet. This droplet was allowed to gelate for about $2 \mathrm{~min}$ resulting in formation of a $20 \mu \mathrm{l}$ hydrogel containing 40,000 cells $\left(2.0 \times 10^{6}\right.$ cells $\left./ \mathrm{ml}\right)$ at a final concentration of 1.50 $\mathrm{mM}$ heparin maleimide conjugate and $1.12 \mathrm{mM}$ starPEG-MMP-peptide conjugate. The gels were then placed in $0.75 \mathrm{ml}$ of $A M$ culture medium per well in 24-well plates, and incubated in 5\% CO2/95\% air at $37^{\circ} \mathrm{C}$

1009 until the desired time points ( 1 week, 2 weeks, and 3 weeks). The 1010 medium was changed 3 times a week throughout the incubation period.

$10112 \mathrm{D}$ pNSC cultures were treated with $2 \mu \mathrm{M} A \beta 42$ in the culture medium for

101248 hours between second and fourth days of culture. The medium is 1013 removed and cells are washed with cell culture medium twice. Amyloid 1014 plaques remain in the culture attached to the cells throughout the 1015 cultures. New medium is added and cultures were continued.

\section{Transplantation}

1017 Primary human neural stem cells (pNSCs) were cultured for two days and 1018 treated with TOPRO-3 (Thermo Scientific) for 1 hour before 1019 transplantation. After a washing step, the cells were harvested with 1020 Accutase and re-suspended in astrocyte cell culture medium at $3 \times 10^{5}$ 1021 cells per $\mathrm{ml}$. With a pipette tip, $2 \mu \mathrm{l}$ of the cell suspension was injected 1022 into the center of the hydrogel. After a week of culture, the hydrogels 1023 were fixed and processed for immunocytochemistry.

\section{Immunocytochemistry}

1025 All of the hydrogels were fixed with ice-cold $4 \%$ paraformaldehyde and 1026 incubated for 1.5 hours at room temperature followed by washing in PBS 1027 overnight at $4{ }^{\circ} \mathrm{C}$. For immunocytochemistry, the hydrogels were blocked 1028 and permeabilized in blocking solution for 4 hours at room temperature. 1029 For BrdU-treatment, the gels were incubated with $2 \mathrm{M} \mathrm{HCl}$ for 20 minutes 1030 at $37^{\circ} \mathrm{C}$ followed by three washes in PBS ( 2 hours each). EdU staining 1031 was performed according to the manufacturer's protocol (Life 
1032 Technologies, C10638) using a 1-hour incubation step. The hydrogels 1033 were incubated with primary antibodies (Supplementary Table 1) in 1034 blocking solution overnight at $4{ }^{\circ} \mathrm{C}$. The gels were washed for two 1035 subsequent days at $4{ }^{\circ} \mathrm{C}$, with occasional changes of the PBS. After 1036 washing, the gels were incubated with the secondary antibodies (1:500 in 1037 blocking solution) at room temperature for 6 hours. After 3 washing steps 1038 of 2 hours each, DAPI staining was performed (1:3000 in PBS, 2 hours at 1039 room temperature). Immunostaining for SOX2 (Santa Cruz Biotechnology, 1040 1:100), SATB2 (Abcam, 1:300), ASCL1 (Neuromics, 1:100), CTIP2 1041 (Abcam, 1:100), EEA1 (Abcam, 1:500), neurofilament (NF-M+H+L) (Life 1042 Technologies, 1:500), TUBB3 (R\&D Systems, 1:500), CASP3 (Santa Cruz 1043 Biotechnology, 1:500), MKI67 (Abcam, 1:1000), acetylated tubulin 1044 (Sigma, 1:500), SYN (Millipore, 1:500), GFAP (Novex, 1:500), DCX 1045 (Novex, 1:300), MAPT (Abcam, 1:500), A 42 (Cell Signaling Technology, 1046 host: Rabbit, 1:500), Reelin (Abcam, 1:500), FOXO1 (Thermo Scientific, 1047 1:500), FOXP2 (R\&D, 1:200), CRYM (Thermo Fischer, 1:50), PSD95 1048 (Thermo Fischer, 1:300), DBX1 (Abcam, 1:300), VGLUT1 (Thermo 1049 Fischer, 1:500), BrdU (AdB Serotec, 1:500) was performed. All of the 1050 secondary antibodies were conjugated to AlexaFluor dyes (Life 1051 Technologies).

\section{Fluorescent imaging}

1053 For the hydrogels, fluorescent imaging was performed using a Leica SP5 1054 inverted Laser Scanning Confocal microscope. The hydrogels were placed 1055 in glass bottom Petri dishes. Sixty microliters of PBS were added on top of 1056 the hydrogels to avoid desiccation. The Z-stacks were captured using a 1057 25x water immersion lens. Every Z-stack had a z-distance of $500 \mu \mathrm{m}$. 1058 Monolayers were imaged using an inverted Zeiss Apotome 2 microscope.

\section{Histological analyses}

1060 For Gallyas silver staining, the 3D hydrogels were cryo-frozen and 1061 sequentially incubated in 5\% periodic acid (5 minutes), an alkaline silver 1062 iodide solution ( 1 minute), acetic acid ( 3 minutes), $0.1 \%$ gold chloride ( 5 1063 minutes), $1 \%$ sodium thiosulfate (5 minutes), and $2.5 \%$ aluminum sulfate 1064 (1 minutes), with intermittent washes with distilled water. For Thioflavin S 
1065 staining, samples were incubated in $1 \%$ Thioflavin S ( 8 minutes), absolute 1066 ethanol (3 minutes), and DAPI (10 minutes).

\section{Transfection with GCaMP6f plasmids and calcium imaging}

1068 TurboFectin 8.0 reagent (OriGene, Cat\# TF81001) was used to transfect 1069 adherent (2D cultures) and encapsulated (3D cultures) cells with $700 \mu \mathrm{g}$ 1070 plasmid per reaction in $1 \mathrm{ml}$ of cell growth medium. The pGP-CMV1071 GCaMP6f plasmid was a gift from Douglas Kim (Addgene plasmid \# 1072 40755)(Chen et al., 2013). The images were captured using a Leica SP5 1073 inverted Laser Scanning Confocal microscope in resonant scanner mode 1074 with photon counting. Images were acquired every 100 milliseconds. 1075 Analysis of the calcium image spectrum was performed with the Leica LAS 1076 AF software by using region of interests (ROIs) and photon counting.

\section{Patch clamp recordings}

1078 Single neurons were recorded in Artificial Cerebrospinal Fluid (ACSF) (119 $1079 \mathrm{mM} \mathrm{NaCl}, 2.5 \mathrm{mM} \mathrm{KCl}, 2 \mathrm{mM} \mathrm{CaCl}, 1.3 \mathrm{mM} \mathrm{MgCl}, 1 \mathrm{mM} \mathrm{NaH} \mathrm{PO}_{4}$, and 10 $1080 \mathrm{mM}$ glucose, $\mathrm{pH}$ 7.3) and patched with nerve solution (125 mM K $\mathrm{K}^{+}$ 1081 gluconate, $0.1 \mathrm{mM} \mathrm{CaCl} 2,0.6 \mathrm{mM} \mathrm{MgCl} 2,8 \mathrm{mM} \mathrm{NaCl}, 1 \mathrm{mM}$ EGTA, 0.01 $1082 \mathrm{mM}$ HEPES, and $4 \mathrm{mM}$ Na-ATP, $\mathrm{pH}$ 7.23) The Whole-cell patch recordings 1083 were assessed using a HEKA set up and Pulse program. Membrane voltage 1084 resistance was held at $-80 \mathrm{mV}$ with the pipette resistance of 4-6 MOhms. 1085 For measurements of $\mathrm{K}+$ and $\mathrm{Na}+$ currents, test pulses were applied in 80 $1086 \mathrm{~ms}$ durations from $-80 \mathrm{mV}$ to $30 \mathrm{mV}$ every $2 \mathrm{~s}$. All experiments were done 1087 at $20-23^{\circ} \mathrm{C}$.

\section{Electron microscopy}

1089 For electron microscopy, the hydrogel-embedded cells were fixed in 1090 modified Karnovsky's fixative (2\% glutaraldehyde $+2 \%$ 1091 paraformaldehyde in $50 \mathrm{mM}$ HEPES) at least overnight at $4{ }^{\circ} \mathrm{C}$. The 1092 samples were washed $2 x$ in 100 mM HEPES and 2x in water and postfixed 1093 in a $2 \%$ aqueous $\mathrm{OsO}_{4}$ solution containing $1.5 \%$ potassium ferrocyanide 1094 and $2 \mathrm{mM} \mathrm{CaCl} 2$ for $30 \mathrm{~min}$ on ice. Next, washes in water, $1 \%$ 1095 thiocarbohydrazide in water (20 minutes at room temperature), water, 1096 and a second osmium contrasting step in $2 \% \mathrm{OsO}_{4} /$ water (30 minutes on 1097 ice). After several washes in water, the samples were en bloc contrasted 
1098 with $1 \%$ uranyl acetate/water for 2 hours on ice, washed again in water, 1099 dehydrated in a graded series of ethanol/water up to $100 \%$ ethanol, and 1100 infiltrated with Epon 812 (Epon/ethanol mixtures: 1:3, 1:1, 3:1 for 1.5 1101 hours each, pure Epon overnight, and pure Epon for 5 hours). The 1102 samples were embedded in flat embedding molds and cured overnight at $110365^{\circ} \mathrm{C}$. Ultrathin sections were prepared with a Leica UC6 ultramicrotome 1104 (Leica Microsystems, Vienna, Austria), collected on Formvar-coated slot 1105 grids and stained with lead citrate and uranyl acetate as previously 1106 described(Venable and Coggeshall, 1965).

1107 For CLEM, cells that were embedded in the hydrogels with the fluorescein1108 labeled peptide were fixed with 4\% paraformaldehyde in $100 \mathrm{mM}$ 1109 phosphate buffer (PB). After several washes in water, the samples were 1110 dehydrated in 50\% (15 minutes at $4{ }^{\circ} \mathrm{C}$ ), $70 \%, 90 \%$, and $100 \%$ acetone 1111 (45 minutes each at $-25^{\circ} \mathrm{C}$ ) and incubated with LR Gold (London Resin 1112 Company, Reading, UK) solutions of $33 \%$ and $66 \%$ LR Gold/acetone, pure 1113 LR Gold ( 1 hour each at $-25^{\circ} \mathrm{C}$ ), and LR Gold $+0.1 \%$ benzil ( 1 hour, 1114 overnight at $-25^{\circ} \mathrm{C}$ ). Finally, the samples were transferred to LR Gold1115 containing $1 \%$ benzil and polymerized using the UV lamp of the Leica 1116 AFS2 freeze substitution unit (Leica Microsystems, Vienna, Austria) for 48 1117 hours at $-25^{\circ} \mathrm{C}$. Ultrathin sections were mounted on Formvar-coated EM 1118 grids, stained with DAPI, imaged with a wide field fluorescence 1119 microscope, washed, and contrasted with 1\% uranyl acetate for EM as 1120 previously described(Fabig et al., 2012). Contrasted ultrathin sections 1121 were analyzed on a FEI Morgagni D268 (FEI, Eindhoven, The Netherlands) 1122 or a Jeol JEM1400 Plus at $80 \mathrm{kV}$ acceleration voltage.

\section{Atomic force microscopy}

1124 Atomic force microscopy (AFM) was performed to determine the 1125 mechanical properties of the gels. Briefly, AFM measurements were 1126 collected at $37^{\circ} \mathrm{C}$ using a Nanowizard II AFM (JPK Instruments, Berlin, 1127 Germany). Tipless silicon nitride cantilevers with a nominal spring 1128 constant of $80 \mathrm{mN}^{*} \mathrm{~m}-1$ (PNP-TR-TL-Au; Nanoworld) were used. The 1129 cantilevers were modified with silica beads ( $110 \mu \mathrm{m}$, Kisker Biotec $1130 \mathrm{GmbH}$ ), as previously described (Bray et al., 2015). Force-distance curves 1131 were acquired in closed loop, constant height mode using a $3 \mathrm{nN}$ contact 
1132 force and a $5 \mu \mathrm{m} / \mathrm{s}$ approach/retract velocity. Each data set was

1133 generated by probing a minimum of 70 different spots on each sample.

1134 The data processing software provided by the AFM manufacturer (JPK 1135 Instruments) was used to extract the Young's Modulus $E$ from the 1136 approach force-distance curves.

\section{RNA Isolation}

1138 RNA isolation from 2D cell culture was performed by TriZol (Invitrogen).

1139 Total RNA isolation from 3D gels was performed by Norgen Total RNA 1140 isolation Kit (Cat\#17200). 5 gels were lysed in $1 \mathrm{ml}$ RL buffer with $10 \mu \mathrm{L}$ $1141 \beta$-mercaptoethanol, and after centrifugation at $12,000 \mathrm{~g}$ for $7 \mathrm{~min}$ at room 1142 temperature, the supernatant was collected in a new Eppendorf and 1143 mixed with absolute Ethanol. The remaining steps performed as 1144 previously described (Bhattarai et al., 2016).

\section{Next generation sequencing of whole transcriptome}

1146 cDNA libraries were prepared by following the protocol for NEBNext® 1147 Ultra I Directional RNA Library Prep Kit. This involves the following steps: 1148 mRNA isolation via poly $(\mathrm{A})+$ selection and fragmentation, first strand and

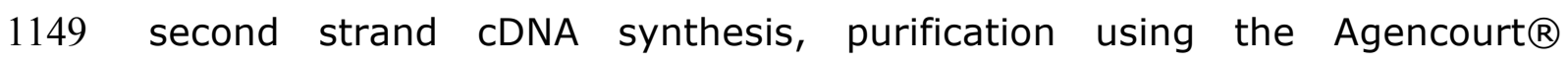

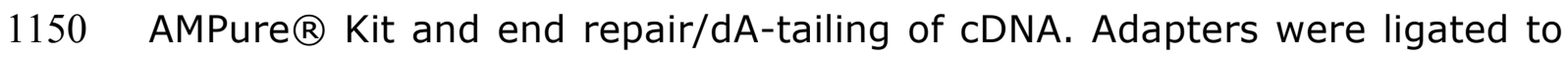
1151 the dA-tailed cDNA, followed by an size selection using AMPure XP Beads.

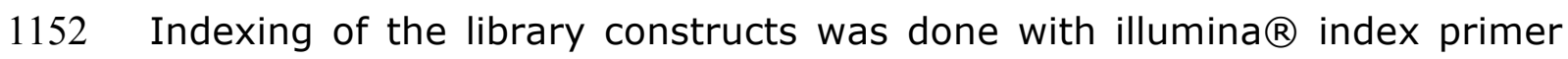
1153 during the following PCR amplification using NEBNext ${ }^{\circledR}$ Q5 2X PCR Master 1154 Mix. Lastly, libraries were purified using the Agencourt $\AA$ AMPure $®$ Kit. 1155 Libraries were pooled and sequenced on an illumina ${ }^{\circledR}$ NextSeq 500 1156 system, resulting in ca. 27 - 38 million 75 bp single-end reads. All 1157 protocols are performed according to the manufacturers' instructions.

\section{Data Analysis}

1159 The reads in fastq files were aligned to the human genome 1160 (hg19/GRCh38) with gsnap (version 2016-09-23) (Wu et al., 2016), and 1161 featureCounts (v1.5.3) (Liao et al., 2013, 2014) was used to assign reads 1162 to each gene using Ensembl version 90 [Homo_sapiens.GRCh38.90.gtf]. 1163 DESeq2 (1.18.0) (Love et al., 2014) was used to normalize the reads, 1164 calculate fold changes and p-values. 2-fold change and padj value of less 
1165 than 0.1 were used to identify differentially expressed genes. For KEGG 1166 pathway analysis GOstats (2.44.0) (Falcon and Gentleman, 2007), GOSeq 1167 (1.30.0) (Young et al., 2010), and clusterProfiler (3.6.0) (Yu et al., 2012) 1168 were used. topGO (2.30.0) (Alexa et al., 2006) was used for GO analysis 1169 and pathview (1.18.0) (Luo and Brouwer, 2013) was used for drawing 1170 KEGG pathway. All data analysis pipeline scripts were written in R in our 1171 lab, and are available upon request.

\section{Sequencing datasets}

1173 All deep sequencing experiments (pNSC 2D, pNSC 3D, iNSC 3D control 1174 and Amyloid-beta42-treated) can be found under the GEO accession 1175 number GSE78117.

\section{Image analysis and statistics}

1177 The 3D reconstructions of hydrogel images and videos were generated 1178 using Arivis 4D software. Images from monolayers were processed using 1179 Zeiss ZEN software. The statistical analyses were performed using 1180 GraphPad Prism and two-tailed Student's t-tests. The levels of significance 1181 were $*: \mathrm{p} \leq 0.05, * *: \mathrm{p} \leq 0.01$, and $* * *: \mathrm{p} \leq 0.001$. In all graphs, means $1182 \pm$ standard deviations are shown.

1183 The effect size was calculated using G-Power, and the sample size was 1184 estimated with n-Query. The data conforms to normal distribution as 1185 determined by Pearson's chi-squared test. The variations between the 1186 samples are similar as determined by variance estimation using Microsoft 1187 Excel software. For 3D gels, 9 gels were used for quantifications (3 1188 technical replicates in every experiment, and 3 experiments as biological 1189 replicates). All experiments were replicated many times in the laboratory 1190 and results were confirmed independently (80-120 gels were qualitatively 1191 analyzed to check the consistency of the results for every individual 1192 experiment).

\section{Generation of skeletonized networks and quantification}

1194 To examine the axons of neural cells, the length and branching were 1195 obtained by thinning binary images to a skeleton, which was performed in 1196 all three dimensions. In detail, the raw images were processed with a 1197 Gaussian filter and then with the tubeness filter to enhance linear 
1198 structures. Then, an automatic threshold was applied, followed by several 1199 morphological operations to facilitate the skeletonization. Fiji software 1200 (www.fiji.sc) was used for image processing. Skeletons were quantified 1201 using KNIME freeware (Supplementary Figure 4). 


\section{Supplementary Information}

\section{Supplementary Figure 1-5}

1204 Supplementary Movies 1-8

1205 Supplementary Dataset 1-12

1206

Supplementary figures
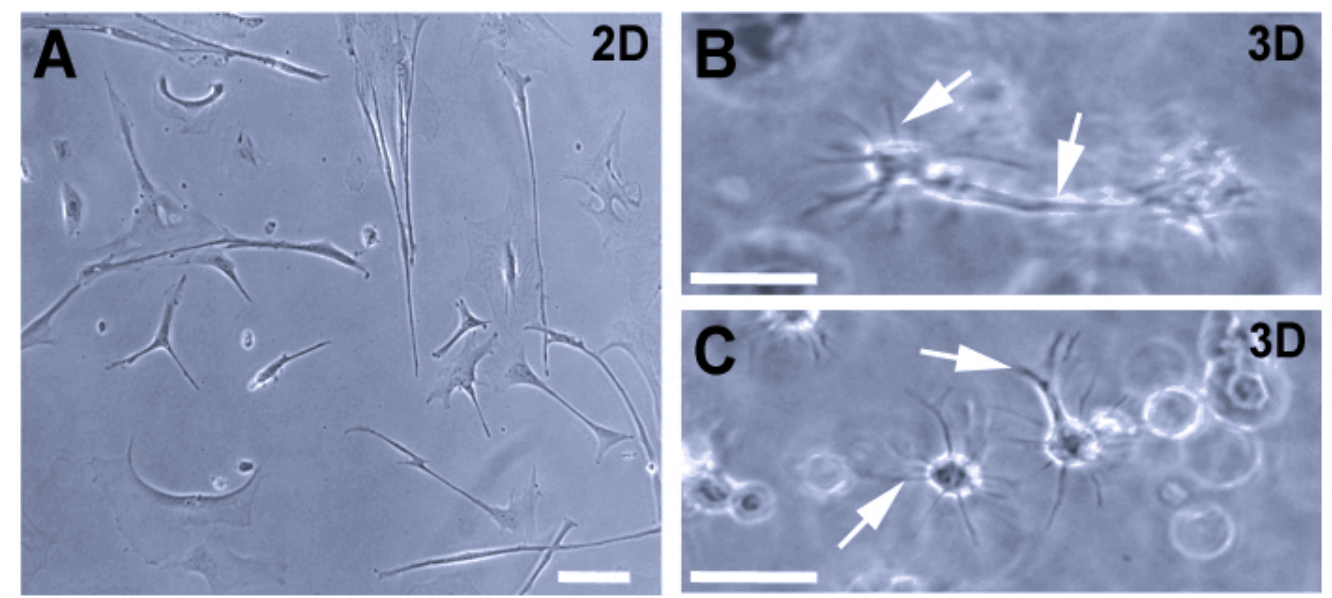

1209 Supplementary Figure 1

1210 Formation of 3D topology and arborizations in starPEG-Heparin gels

1212 (A) Culture of primary human NSCs in 2D.

$1213(B, C)$ Culture of primary human NSCs in 3D. Note the arborized

1214 morphology and cellular processes reminiscent of in vivo (white arrows).

1215 Scale bars $25 \mu \mathrm{m}$. Related to Figure 1.

1216 


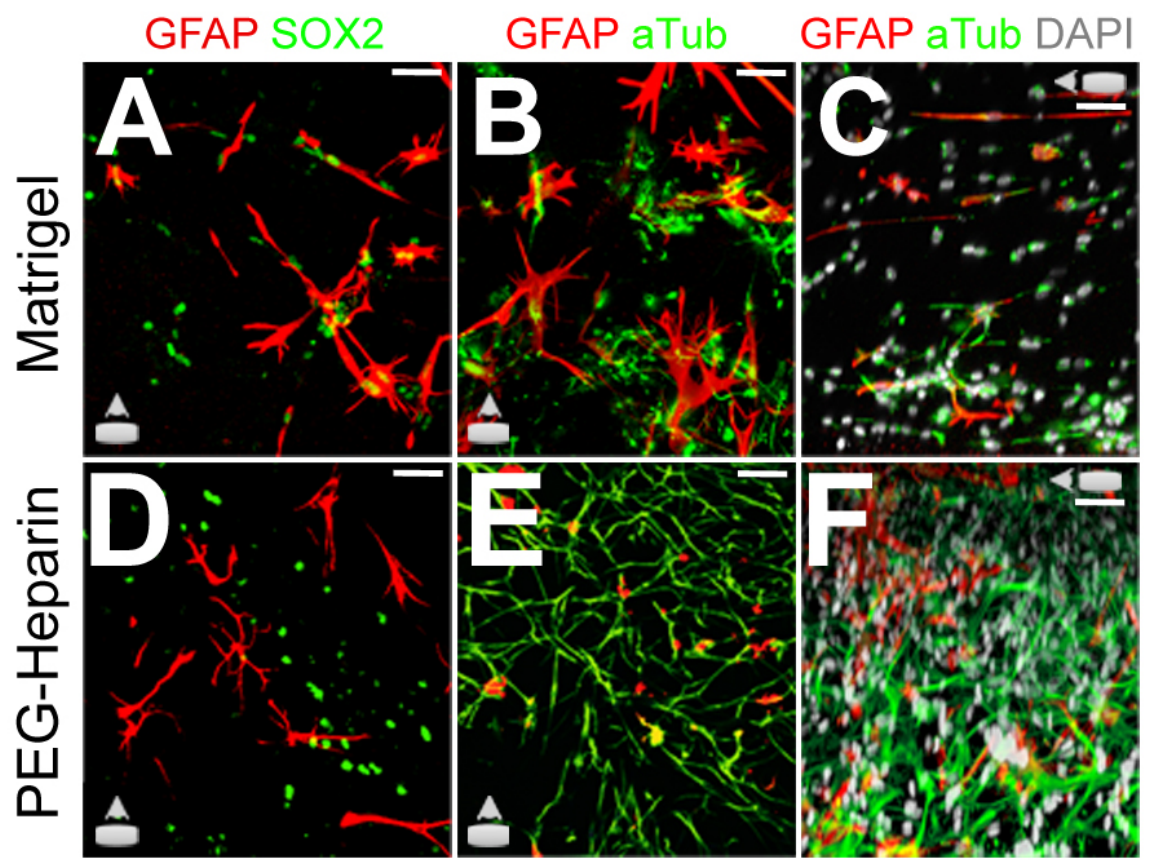

1218 Supplementary Figure 2

1219 Comparison of Matrigel and starPEG-Heparin cultures

1221 (A) Immunostaining for GFAP and SOX2 in Matrigel cultures.

1222 (B) Immunostaining GFAP and Acetylated tubulin in Matrigel cultures Z1223 view.

1224 (C) Immunostaining GFAP and Acetylated tubulin in Matrigel cultures X1225 view.

1226 (D) Immunostaining for GFAP and SOX2 in starPEG-Heparin cultures.

1227 (E) Immunostaining GFAP and Acetylated tubulin in starPEG-Heparin 1228 cultures Z-view.

1229 (F) Immunostaining GFAP and Acetylated tubulin in starPEG-Heparin 1230 cultures $X$-view.

1231 Scale bars: $20 \mu \mathrm{m}$. All gels are 3 weeks of culture. 


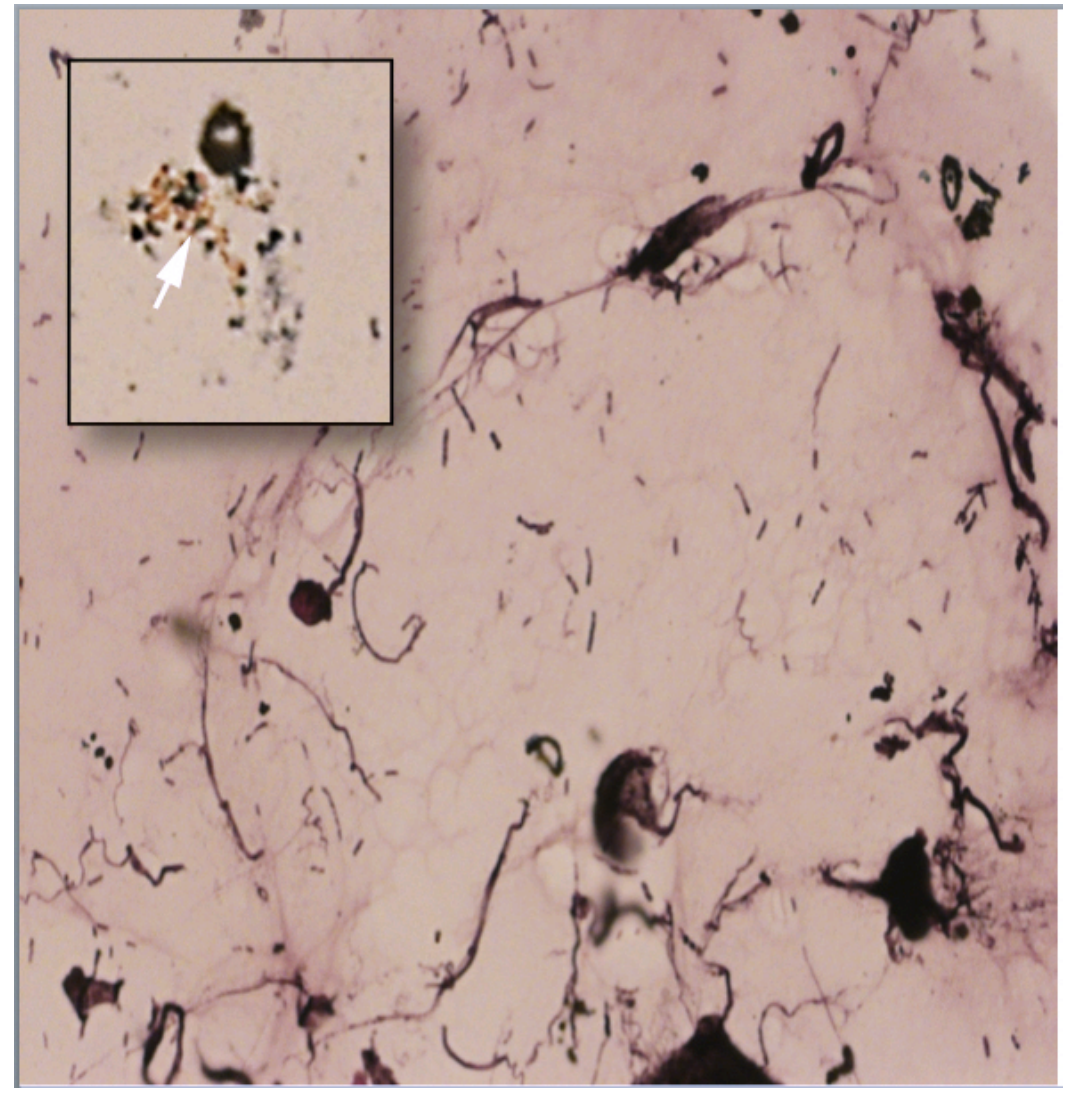

1234 Supplementary Figure 3

1235 Formation of neurofibrillary tangles and senile plaques in 3D cultures of 1236 primary human NSCS.

1238 Image shows Gallyas silver impregnation staining for neurofibrillary

1239 tangles in 3D cultures. Inset shows the senile plaques (white arrow). 


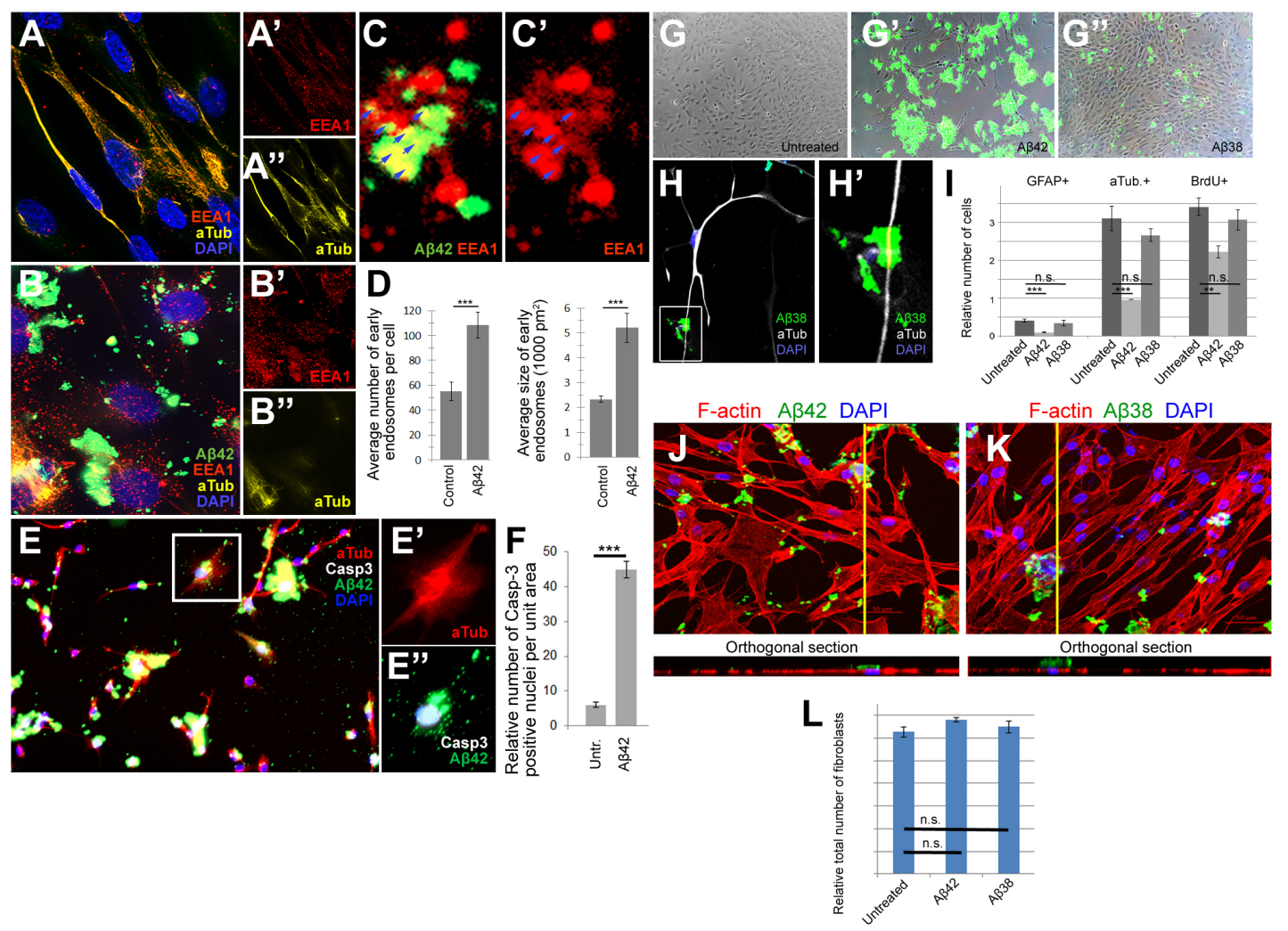

\section{Supplementary Figure 4}

1243 Amyloid aggregation dynamics

(A) Immunostaining for acetylated tubulin and early endosomes (EEA1) in 1246 control cultures.

$1247\left(A^{\prime}, A^{\prime \prime}\right)$ Individual fluorescence channels for EEA1 and acetylated tubulin 1248 in control cultures.

1249 (B) Immunostaining for acetylated tubulin and early endosomes (EEA1) in 1250 A 4 42-treated cultures.

1251 ( $\left.\mathrm{B}^{\prime}, \mathrm{B}^{\prime \prime}\right)$ Individual fluorescence channels for EEA1 and acetylated tubulin 1252 in A 42 -treated PHCCs.

1253 (C, C') Co-localization of EEA1 and $A \beta 42$.

1254 (D) Quantification of the average number of early endosomes per cell and 1255 the average size of early endosomes per cell in control and Aß42-treated 1256 cultures.

1257 (E) Immunostaining of Aß42-treated cells for acetylated tubulin (red), 1258 A 42 (green) and caspase-3 (white).

1259 ( $\left.E^{\prime}\right)$ Individual fluorescence channel for acetylated tubulin.

1260 ( $\left.E^{\prime \prime}\right)$ Fluorescence channels for $A \beta 42$ and Caspase-3. 
1261 (F) Quantification of Caspase-3-positive cells in control and Aß42-treated 1262 cultures.

1263 (G) Bright field image of control cultures.

$1264\left(G^{\prime}\right)$ Bright field image of $A \beta 42$-treated cultures.

$1265\left(G^{\prime \prime}\right)$ Bright field image of Aß38-treated cultures.

$1266(\mathrm{H})$ Confocal image of acetylated tubulin immunostaining on A 338 -treated 1267 cultures.

$1268\left(\mathrm{H}^{\prime}\right)$ Close-up of a region from $\mathrm{H}$ showing cells treated with $A \beta 38$.

1269 (I) Quantification of the relative number of untreated, $A \beta 42$-treated and 1270 A 338 -treated cells immunoreactive for GFAP, acetylated tubulin or BrdU.

1271 (J) Confocal image over the $z$-axis and orthogonal section over the $y$-axis

1272 of A 442 -treated human-derived fibroblasts. F-actin was stained with 1273 phalloidin, and DNA was stained with DAPI.

1274 (K) Confocal image over the $z$-axis and orthogonal section over the $y$-axis

1275 of Aß38-treated human-derived fibroblasts. F-actin was stained with 1276 phalloidin, and DNA was stained with DAPI.

1277 (L) Quantification of the relative total number of fibroblasts in control, 1278 A 4 42-treated and $A \beta 38$-treated samples.

1279 Scale bars $20 \mu \mathrm{m}$. Related to Figure 4. 
bioRxiv preprint doi: https://doi.org/10.1101/225243; this version posted November 27, 2017. The copyright holder for this preprint (which was not certified by peer review) is the author/funder. All rights reserved. No reuse allowed without permission.

1 title = getTitle();

2 dir = getDirectory ("image");

3 getVoxelsize(width, height, depth, unit);

4 run("Duplicate...", "title=Duplicate duplicate channels=2");

5 selectWindow(title);

6 close ();

7 selectWindow("Duplicate");

8 run("Gaussian Blur...", "sigma=0.85 stack");

9 run("Tubeness", "sigma=3");

10 setAutoThreshold("Triangle dark stack");

11 setoption("BlackBackground", false);

12 run("Convert to Mask", "method=Triangle background=Dark black");

13 run("Invert LUT");

14 run("Options...", "iterations=6 count=3 pad do=Close");

15 run("Erode (3D)", "iso=255");

16 run("Dilate (3D)", "iso=255");

17 run("Dilate (3D)", "iso=255");

18 run("Erode (3D)", "iso=255");

19 run("Skeletonize (2D/3D)");

20 setvoxelsize(width, height, depth, unit);

21 run("Analyze Skeleton (2D/3D)", "prune=none calculate");

22 newtitle = substring(title,0,lastIndexof(title, ".lif"));

23 saveAs("results", dir+newtitle+".xls");

24 selectwindow("Tagged skeleton");

25 saveAs("Tiff", dir+newtitle+"_skel.tif");

$26 / /$ run ("Close All");

\section{Supplementary Figure 5}

1283 Script for KNIME software

1284

1285

1286

1287 
1288

1289

1290

1291

1292

1293

1294

1295

1296

1297

1298

1299

1300

1301

1302

1303

1304

1305

1306

1307

1308

1309

1310

1311

1312

1313

1314

1315

1316

1317

1318

1319

1320

1321

1322

\section{Supplementary Movies}

\section{Supplementary Movie 1}

Neuronal network in pNSC cultures

$3 \mathrm{D}$ representation of a $0.18 \mathrm{~mm}^{3}$ portion of a control hydrogel containing networks of neurons as assessed by acetylated tubulin staining (cyan). Related to Figure 1.

\section{Supplementary Movie 2}

Neuronal network in iNSC cultures

3D reconstruction of iPSC-derived NSC cultures in PEG-Heparin gels. Cyan: acetylated tubulin, white: DAPI, red: GFAP. Related to Figure 1.

\section{Supplementary Movie 3}

\section{Calcium activity}

Encapsulated primary human cortical astrocytes in PEG-HEP gels transfected with the GCaMP6f Calcium sensor exhibit green fluorescence in response to calcium influx after treatment with glutamate. Related to Figure 1.

\section{Supplementary Movie 4}

\section{Dystrophic axons}

A TUBB3-positive neuron (cyan) with retracted processes, dystrophic axonal ends, and $A \beta 42$ aggregation (green) is shown in a 3D reconstruction. DAPI is shown in white. Related to Figure 2.

\section{Supplementary Movie 5}

$3 D$ reconstruction of a neuron with hyperphosphorylated Tau

A 3D reconstruction of primary human cortical astrocyte cultures after 3 weeks in $A \beta 42$-treated gels and staining for $A \beta 42$ (green), DAPI (white) 
1323 and phosphorylated Tau (AT180, red). Note that neurons exhibit staining

1324 for hyperphosphorylated Tau. Related to Figure 2.

1325

1326

\section{Supplementary Movie 6}

Transplantation in control gels

3D reconstruction of a labeled neuron (red nuclei, transplanted) that

1330 established connections to the existing neurons in the control gels. Cyan:

1331 acetylated tubulin, white: DAPI, red: TOPRO3. Related to Figure 2.

\section{Supplementary Movie 7}

1334 Transplantation in Amyloid-containing gel

1336 3D reconstruction of a labeled neuron (red nuclei, transplanted) that was

1337 unable to make connections with the existing, already dystrophic, neurons

1338 in amyloid-containing gels. Green: Aß42, cyan: acetylated tubulin, white:

1339 DAPI, red: TOPRO3. Related to Figure 2.

\section{Supplementary Movie 8}

1342 Comparison of gel preparation

1344 Comparison of preparation of starPEG-Heparin and Matrigel cultures of 1345 primary human astrocytes. Related to Figure 2. 


\section{Supplementary Datasets}

1351

\section{2}

\section{Supplementary Dataset 1}

1353 Differential expression analyses between pNSC 3D cultures and pNSC 2D

1354 cultures.

\section{Supplementary Dataset 2}

1357 Elim Fischer cellular component analyses of differentially expressed genes 1358 between pNSC 3D cultures and pNSC 2D cultures.

\section{Supplementary Dataset 3}

1361 KEGG pathway analysis of differentially expressed genes between pNSC 1362 3D cultures and pNSC 2D cultures.

\section{Supplementary Dataset 4}

1364 Differential expression analyses between iNSC 3D cultures and pNSC 3D 1365 cultures.

\section{Supplementary Dataset 5}

1368 Elim Fischer cellular component analyses of differentially expressed genes between iNSC 3D cultures and pNSC 3D cultures.

\section{Supplementary Dataset 6}

1372 KEGG pathway analysis of differentially expressed genes between iNSC 3D cultures and pNSC 3D cultures.

\section{Supplementary Dataset 7}

1376 Differential expression analyses upon A 42 in 3D iNSC cultures.

\section{Supplementary Dataset 8}

1379 Differential expression analyses upon A 42 in 3D pNSC cultures.

\section{Supplementary Dataset 9}

1382 Elim Fischer cellular component analyses of differentially expressed genes

1383 in Aß42-treated 3D iNSC cultures. 


\section{Supplementary Dataset 10}

1386 KEGG pathway analysis of differentially expressed genes in Aß42-treated 1387 3D iNSC cultures.

1388

\section{Supplementary Dataset 11}

1390 Elim Fischer cellular component analyses of differentially expressed genes 1391 in Aß42-treated 3D pNSC cultures.

\section{Supplementary Dataset 12}

1394 KEGG pathway analysis of differentially expressed genes in Aß42-treated 1395 3D pNSC cultures. 